\title{
ANALYSIS OF ELECTROELASTIC FRICTIONLESS CONTACT PROBLEMS WITH ADHESION
}

\author{
MIRCEA SOFONEA, RACHID ARHAB, AND RAAFAT TARRAF \\ Received 24 January 2006; Revised 29 May 2006; Accepted 5 June 2006
}

We consider two quasistatic frictionless contact problems for piezoelectric bodies. For the first problem the contact is modelled with Signorini's conditions and for the second one is modelled with normal compliance. In both problems the material's behavior is electroelastic and the adhesion of the contact surfaces is taken into account and is modelled with a surface variable, the bonding field. We provide variational formulations for the problems and prove the existence of a unique weak solution to each model. The proofs are based on arguments of time-dependent variational inequalities, differential equations, and fixed point. Moreover, we prove that the solution of the Signorini contact problem can be obtained as the limit of the solution of the contact problem with normal compliance as the stiffness coefficient of the foundation converges to infinity.

Copyright (c) 2006 Mircea Sofonea et al. This is an open access article distributed under the Creative Commons Attribution License, which permits unrestricted use, distribution, and reproduction in any medium, provided the original work is properly cited.

\section{Introduction}

Considerable progress has been achieved recently in modelling, mathematical analysis, and numerical simulations of various contact processes and, as a result, a general mathematical theory of contact mechanics is currently emerging. It is concerned with the mathematical structures which underlie general contact problems with different constitutive laws, that is, materials, varied geometries, and different contact conditions, see, for instance, $[14,20]$ and the references therein; its aim is to provide a sound, clear, and rigorous background to the constructions of models for contact, proving existence, uniqueness, and regularity results, assigning precise meaning to solutions, among others.

In this paper we study two quasistatic frictionless contact problems for electroelastic materials with adhesion, in the framework of the mathematical theory of contact mechanics; our interest is to describe two physical processes in which both contact, adhesion and piezoelectric effect are involved, to show that the resulting models lead to well-posedness mathematical problems, and to study the link between the processes by

Hindawi Publishing Corporation

Journal of Applied Mathematics

Volume 2006, Article ID 64217, Pages 1-25

DOI 10.1155/JAM/2006/64217 
proving a convergence result. Taking into account the piezoelectric effect in the study of quasistatic adhesive contact problems represents the main trait of novelty of this paper.

The piezoelectric effect is characterized by the coupling between the mechanical and electrical properties of the materials. Indeed, the apparition of electric charges on some crystals submitted to the action of body forces and surface tractions was observed and their dependence on the deformation process was underlined. Conversely, it was proved experimentally that the action of electric field on the crystals may generate strain and stress. A deformable material which presents such a behavior is called a piezoelectric material. Piezoelectric materials are used extensively as switches and actuary in many engineering systems, in radioelectronics, electroacoustics, and measuring equipments. General models for electroelastic materials can be found in [3, 5, 15]. A static frictional contact problem for electroelastic materials was considered in [4, 16]. A slip-dependent frictional contact problem for electroelastic materials was studied in [21] and a frictional problem with normal compliance for electroviscoelastic materials was considered in [22]. In the last two references the variational formulations of the corresponding problems were derived and existence and uniqueness results for the weak solutions were obtained.

The adhesive contact between deformable bodies, when a glue is added to prevent relative motion of the surfaces, has received recently increased attention in the mathematical literature. Basic modelling can be found in $[10-12,17,25]$. Analysis of models for adhesive contact can be found in $[1,2,6-9,13,24]$ and in the recent monographs $[20,23]$. An application of the theory of adhesive contact in the medical field of prosthetic limbs was considered in $[18,19]$; there, the importance of the bonding between the bone-implant and the tissue was outlined, since debonding may lead to decrease in the persons ability to use the artificial limb or joint. The novelty in all the above papers is the introduction of a surface internal variable, the bonding field, denoted in this paper by $\beta$; it describes the pointwise fractional density of active bonds on the contact surface, and sometimes referred to as the intensity of adhesion. Following $[10,11]$, the bonding field satisfies the restrictions $0 \leq \beta \leq 1$; when $\beta=1$ at a point of the contact surface, the adhesion is complete and all the bonds are active; when $\beta=0$, all the bonds are inactive, severed, and there is no adhesion; when $0<\beta<1$, the adhesion is partial and only a fraction $\beta$ of the bonds is active. We refer the reader to the extensive bibliography on the subject in $[12,17,18,20,23]$.

For the first problem we study in this paper the contact is modelled with Signorini's conditions and for the second one is modelled with normal compliance. In both problems the contact is adhesive and the piezoelectric effect is taken into account. We derive a variational formulation of the problems and prove the existence of a unique weak solution to each one. To this end we use similar arguments as in $[6,7,9,24]$ but with a different choice of functionals and operators, since the constitutive law and the contact boundary conditions, here and in the above-mentioned papers, is different. Moreover, we study the behavior of the solution of the problem with normal compliance as the stiffness coefficient of the foundation converges to infinity. Most of the results presented in this paper can be extended to the case of multibody contact.

The paper is structured as follows. In Section 2 we present the models of electroelastic frictionless contact with adhesion and provide comments on the contact boundary 
conditions. In Section 3 we list the assumptions on the data, derive the variational formulation of each model, and state our main results, Theorems 3.4-3.6. Theorems 3.4 and 3.5 state the unique weak solvability of the adhesive frictionless contact problem with Signorini and normal compliance conditions, respectively. Their proofs are provided in Section 4 and are based on arguments of nonlinear equations with monotone operators, differential equations and fixed point. Theorem 3.6 states the convergence of the solution of the electroelastic adhesive contact problem with normal compliance to the solution of the electroelastic adhesive Signorini contact problem, as the stiffness coefficient of the foundation converges to infinity. Its proof is provided in Section 5 and is based on monotonicity, lower semicontinuity, and compactness arguments.

\section{Problems statement}

We consider the following physical setting. An electroelastic body occupies a bounded domain $\Omega \subset \mathbb{R}^{d}(d=2,3)$ with a smooth boundary $\partial \Omega=\Gamma$. The body is submitted to the action of body forces of density $\mathbf{f}_{0}$ and volume electric charges of density $q_{0}$. It is also submitted to mechanical and electric constraints on the boundary. To describe them, we consider a partition of $\Gamma$ into three measurable parts $\Gamma_{1}, \Gamma_{2}, \Gamma_{3}$, on one hand, and on two measurable parts $\Gamma_{a}$ and $\Gamma_{b}$, on the other hand, such that meas $\Gamma_{1}>0$, meas $\Gamma_{a}>0$, and $\Gamma_{3} \subseteq \Gamma_{b}$. We assume that the body is clamped on $\Gamma_{1}$ and surface tractions of density $\mathbf{f}_{2}$ act on $\Gamma_{2}$. On $\Gamma_{3}$ the body is in adhesive contact with an insulator obstacle, the so-called foundation. We also assume that the electrical potential vanishes on $\Gamma_{a}$ and a surface electric charge of density $q_{2}$ is prescribed on $\Gamma_{b}$. We denote by $\mathbb{S}^{d}$ the space of secondorder symmetric tensors on $\mathbb{R}^{d}$ and we use $\cdot$ and $\|\cdot\|$ for the inner product and the Euclidean norm on $\mathbb{R}^{d}$ and $\mathbb{S}^{d}$, respectively. Also, below $\boldsymbol{v}$ represents the unit outward normal on $\Gamma$.

For the first problem we consider here the contact is modeled with Signorini's conditions with adhesion. Thus, the classical model for the process is the following.

Problem 2.1. Find a displacement field $\mathbf{u}: \Omega \times[0, T] \rightarrow \mathbb{R}^{d}$, a stress field $\boldsymbol{\sigma}: \Omega \times[0$, $T] \rightarrow \mathbb{S}^{d}$, an electric potential $\varphi: \Omega \times[0, T] \rightarrow \mathbb{R}$, an electric displacement field $\mathbf{D}: \Omega \times$ $[0, T] \rightarrow \mathbb{R}^{d}$, and a bonding field $\beta: \Omega \times[0, T] \rightarrow \mathbb{R}$ such that

$$
\begin{gathered}
\boldsymbol{\sigma}=\mathscr{A} \boldsymbol{\varepsilon}(\mathbf{u})-\mathscr{E} * \mathbf{E}(\varphi) \quad \text { in } \Omega \times(0, T), \\
\mathbf{D}=\mathscr{B} \mathbf{E}(\varphi)+\mathscr{E} \boldsymbol{\varepsilon}(\mathbf{u}) \quad \text { in } \Omega \times(0, T), \\
\operatorname{Div} \boldsymbol{\sigma}+\mathbf{f}_{0}=\mathbf{0} \quad \text { in } \Omega \times(0, T), \\
\operatorname{div} \mathbf{D}=q_{0} \quad \text { in } \Omega \times(0, T), \\
\mathbf{u}=\mathbf{0} \quad \text { on } \Gamma_{1} \times(0, T), \\
\boldsymbol{\sigma} \boldsymbol{\nu}=\mathbf{f}_{2} \quad \text { on } \Gamma_{2} \times(0, T), \\
u_{\nu} \leq 0, \quad \sigma_{\nu}-\gamma_{\nu} R_{\nu}\left(u_{\nu}\right) \beta^{2} \leq 0, \\
\left(\sigma_{\nu}-\gamma_{\nu} R_{\nu}\left(u_{\nu}\right) \beta^{2}\right) u_{\nu}=0 \quad \text { on } \Gamma_{3} \times(0, T), \\
-\sigma_{\tau}=p_{\tau}(\beta) \mathbf{R}_{\tau}\left(\mathbf{u}_{\tau}\right) \quad \text { on } \Gamma_{3} \times(0, T),
\end{gathered}
$$




$$
\begin{gathered}
\dot{\beta}=-\left(\beta\left(\gamma_{\nu} R_{\nu}\left(u_{\nu}\right)^{2}+\gamma_{\tau}\left\|\mathbf{R}_{\tau}\left(\mathbf{u}_{\tau}\right)\right\|^{2}\right)-\epsilon_{a}\right)_{+} \quad \text { on } \Gamma_{3} \times(0, T), \\
\varphi=0 \quad \text { on } \Gamma_{a} \times(0, T), \\
\mathbf{D} \cdot \boldsymbol{v}=q_{2} \quad \text { on } \Gamma_{b} \times(0, T), \\
\beta(0)=\beta_{0} \quad \text { on } \Gamma_{3} .
\end{gathered}
$$

We now provide some comments on equations and conditions (2.1)-(2.12) and send to $[20,23]$ for more details on the conditions $(2.7)-(2.9)$ which describe the frictionless contact with adhesion.

First, (2.1) and (2.2) represent the electroelastic constitutive law in which $\boldsymbol{\varepsilon}(\mathbf{u})$ denotes the linearized strain tensor, $\mathrm{E}(\varphi)=-\nabla \varphi$ is the electric field, $\mathscr{A}$ is the elasticity operator, $\mathscr{E}$ represents the piezoelectric operator, $\mathscr{E}^{*}$ is its transposed, and $\mathscr{B}$ denotes the electric permittivity operator. Details on the constitutive equations of the form (2.1), (2.2) can be found, for instance, in [3,4]. Next, (2.3) and (2.4) are the equilibrium equations for the stress and electric-displacement fields, respectively, in which "Div" and "div" denote the divergence operator for tensor- and vector-valued functions, respectively. Conditions (2.5) and (2.6) are the displacement and traction boundary conditions, whereas (2.10) and (2.11) represent the electric boundary conditions.

Conditions (2.7) represent the Signorini conditions with adhesion, where $u_{v}$ is the normal displacement, $\sigma_{\nu}$ represents the normal stress, $\gamma_{\nu}$ denotes a given adhesion coefficient, and $R_{\nu}$ is the truncation operator defined by

$$
R_{\nu}(s)= \begin{cases}L & \text { if } s<-L \\ -s & \text { if }-L \leq s \leq 0 \\ 0 & \text { if } s>0\end{cases}
$$

Here $L>0$ is the characteristic length of the bond, beyond which it does not offer any additional traction. The introduction of the operator $R_{\nu}$, together with the operator $\mathbf{R}_{\tau}$ defined below, is motivated by the mathematical arguments but it is not restrictive for physical point of view, since no restriction on the size of the parameter $L$ is made in what follows. Thus, by choosing $L$ very large, we can assume that $R_{\nu}\left(u_{v}\right)=-u_{v}$ and, therefore, from (2.7) we recover the contact conditions

$$
u_{v} \leq 0, \quad \sigma_{\nu}+\gamma_{\nu} u_{v} \beta^{2} \leq 0, \quad\left(\sigma_{\nu}+\gamma_{\nu} u_{\nu} \beta^{2}\right) u_{v}=0 \quad \text { on } \Gamma_{3} \times(0, T) .
$$

These conditions were used in $[8,17]$ to model the unilateral adhesive contact. It follows from (2.7) that there is no penetration between the body and the foundation, since $u_{v} \leq 0$ during the process. Also, note that when the bonding field vanishes, then the contact conditions (2.7) become the classical Signorini contact conditions in the form with a zero gap function, that is,

$$
u_{\nu} \leq 0, \quad \sigma_{\nu} \leq 0, \quad \sigma_{\nu} u_{\nu}=0 \quad \text { on } \Gamma_{3} \times(0, T) .
$$

Condition (2.8) represents the adhesive contact condition on the tangential plane in which $\mathbf{u}_{\tau}$ is the tangential displacement, $\boldsymbol{\sigma}_{\tau}$ represents the tangential stress, $p_{\tau}$ denotes a 
given function, and $\mathbf{R}_{\tau}$ is the truncation operator given by

$$
\mathbf{R}_{\tau}(\mathbf{v})= \begin{cases}\mathbf{v} & \text { if }\|\mathbf{v}\| \leq L, \\ L \frac{\mathbf{v}}{\|\mathbf{v}\|} & \text { if }\|\mathbf{v}\|>L .\end{cases}
$$

This condition shows that the magnitude of the shear on the contact surface depends on the bonding field and on the tangential displacement, but as long as it does not exceed the bond length $L$. The frictional tangential traction is assumed to be much smaller than the adhesive one and, therefore, omitted.

Equation (2.9) describes the evolution of the bonding field with given positive material parameters $\gamma_{\nu}, \gamma_{\tau}$, and $\epsilon_{a}$, in which $r_{+}=\max \{r, 0\}$. Recall that $\epsilon_{a}$ represents the so-called Duprés surface energy and it measures the amount of energy needed to debond a unit of surface area, see [12] for details. Here and below in this paper the dot above represents the derivative with respect to the time variable. We note that the adhesive process is irreversible and, indeed, once debonding occurs, bonding cannot be reestablished, since $\dot{\beta} \leq 0$.

Finally, (2.12) represents the initial condition in which $\beta_{0}$ is a given bonding field.

For the second problem we study in this paper the contact is modeled with normal compliance and adhesion, and therefore the classical model for the process is the following.

Problem 2.2. Find a displacement field $\mathbf{u}_{\mu}: \Omega \times[0, T] \rightarrow \mathbb{R}^{d}$, a stress field $\boldsymbol{\sigma}_{\mu}: \Omega \times[0, T] \rightarrow$ $\mathbb{S}^{d}$, an electric potential $\varphi_{\mu}: \Omega \times[0, T] \rightarrow \mathbb{R}$, an electric displacement field $\mathbf{D}_{\mu}: \Omega \times[0$, $T] \rightarrow \mathbb{R}^{d}$, and a bonding field $\beta_{\mu}: \Omega \times[0, T] \rightarrow \mathbb{R}$ such that

$$
\begin{gathered}
\boldsymbol{\sigma}_{\mu}=\mathscr{A} \boldsymbol{\varepsilon}\left(\mathbf{u}_{\mu}\right)-\mathscr{E} * \mathbf{E}\left(\varphi_{\mu}\right) \quad \text { in } \Omega \times(0, T), \\
\mathbf{D}_{\mu}=\mathscr{B} \mathbf{E}\left(\varphi_{\mu}\right)+\mathscr{E} \boldsymbol{\varepsilon}\left(\mathbf{u}_{\mu}\right) \quad \text { in } \Omega \times(0, T), \\
\operatorname{Div} \boldsymbol{\sigma}_{\mu}+\mathbf{f}_{0}=\mathbf{0} \quad \text { in } \Omega \times(0, T), \\
\operatorname{div} \mathbf{D}_{\mu}=q_{0} \quad \text { in } \Omega \times(0, T), \\
\mathbf{u}_{\mu}=\mathbf{0} \quad \text { on } \Gamma_{1} \times(0, T), \\
\boldsymbol{\sigma}_{\mu} \boldsymbol{\nu}=\mathbf{f}_{2} \quad \text { on } \Gamma_{2} \times(0, T), \\
-\sigma_{\mu \nu}=\frac{1}{\mu} p_{\nu}\left(u_{\mu \nu}\right)-\gamma_{\nu} \beta_{\mu}^{2} R_{\nu}\left(u_{\mu \nu}\right) \quad \text { on } \Gamma_{3} \times(0, T), \\
-\boldsymbol{\sigma}_{\mu \tau}=p_{\tau}(\beta) \mathbf{R}_{\tau}\left(\mathbf{u}_{\mu \tau}\right) \quad \text { on } \Gamma_{3} \times(0, T), \\
\beta_{\mu}=-\left(\beta\left(\gamma_{\mu \nu} R_{\nu}\left(u_{\mu \nu}\right)^{2}+\gamma_{\tau}\left\|\mathbf{R}_{\tau}\left(\mathbf{u}_{\mu \tau}\right)\right\|^{2}\right)-\epsilon_{a}\right) \\
\varphi_{\mu}=0 \quad \text { on } \Gamma_{a} \times(0, T), \\
\mathbf{D}_{\mu} \cdot \boldsymbol{v}=q_{2} \quad \text { on } \Gamma_{b} \times(0, T), \\
\beta_{\mu}(0)=\beta_{0} \quad \text { on } \Gamma_{3} .
\end{gathered}
$$


Here and below $u_{\mu \nu}$ and $\mathbf{u}_{\mu \tau}$ represent the normal and tangential components of the displacement field $\mathbf{u}_{\mu}$; also, $\sigma_{\mu \nu}$ and $\sigma_{\mu \tau}$ represent the normal and tangential components of the stress vector field $\boldsymbol{\sigma}_{\mu} \boldsymbol{\nu}$.

Note that equations and conditions involved in Problem 2.2 have the same significance as those involved in Problem 2.1. The difference arises from the fact that here we replace Signorini's contact conditions with adhesion, (2.7), with the normal compliance contact condition with adhesion, (2.23), where $p_{\nu}$ is a given positive function which will be described below and $\mu>0$ is a penalization parameter which may be interpreted as the deformability coefficient of the foundation, and then $1 / \mu$ is the surface stiffness coefficient. Indeed, when $\mu$ is smaller, the reaction force of the foundation to penetration is larger, and so the same force will result in a smaller penetration, which means that the foundation is less deformable. When $\mu$ is larger, the reaction force of the foundation to penetration is smaller, and so the foundation is less stiff and more deformable. In condition (2.23) the inter-penetrability between the body and the foundation is allowed, that is, $u_{\mu \nu}$ can be positive on $\Gamma_{3}$. The contribution of the adhesive to the normal traction is represented by the term $\gamma_{\nu} \beta_{\mu}^{2} R_{\nu}\left(u_{\mu \nu}\right)$; thus, the adhesive traction is tensile and is proportional, with proportionality coefficient $\gamma_{\nu}$, to the square of the intensity of adhesion and to the normal displacement, but as long as it does not exceed the bond length $L$.

\section{Variational formulations and main results}

In this section we list the assumptions on the data, derive a variational formulation for the contact problems, and state our main results, Theorems 3.4-3.6. To this end we need to introduce some notation and preliminary material.

We recall that the inner products and the corresponding norms on $\mathbb{R}^{d}$ and $\mathbb{S}^{d}$ are given by

$$
\begin{aligned}
& \mathbf{u} \cdot \mathbf{v}=u_{i} v_{i}, \quad\|\mathbf{v}\|=(\mathbf{v} \cdot \mathbf{v})^{1 / 2} \quad \forall \mathbf{u}, \mathbf{v} \in \mathbb{R}^{d}, \\
& \boldsymbol{\sigma} \cdot \boldsymbol{\tau}=\sigma_{i j} \boldsymbol{\tau}_{i j}, \quad\|\boldsymbol{\tau}\|=(\boldsymbol{\tau} \cdot \boldsymbol{\tau})^{1 / 2} \quad \forall \boldsymbol{\sigma}, \boldsymbol{\tau} \in \mathbb{S}^{d} .
\end{aligned}
$$

Here and everywhere in this paper $i, j, k, l$ run from 1 to $d$, summation over repeated indices is implied, and the index that follows a comma represents the partial derivative with respect to the corresponding component of the spatial variable, for example, $u_{i, j}=$ $\partial u_{i} / \partial x_{j}$

Everywhere below we use the classical notation for $L^{p}$ and Sobolev spaces associated to $\Omega$ and $\Gamma$. Moreover, we use the notation $L^{2}(\Omega)^{d}, H^{1}(\Omega)^{d}$, $\mathscr{H}$, and $\mathscr{H}_{1}$ for the following spaces:

$$
\begin{gathered}
L^{2}(\Omega)^{d}=\left\{\mathbf{v}=\left(v_{i}\right) \mid v_{i} \in L^{2}(\Omega)\right\}, \\
H^{1}(\Omega)^{d}=\left\{\mathbf{v}=\left(v_{i}\right) \mid v_{i} \in H^{1}(\Omega)\right\}, \\
\mathscr{H}=\left\{\boldsymbol{\tau}=\left(\tau_{i j}\right) \mid \tau_{i j}=\tau_{j i} \in L^{2}(\Omega)\right\}, \\
\mathscr{H}_{1}=\left\{\boldsymbol{\tau} \in \mathscr{H} \mid \tau_{i j, j} \in L^{2}(\Omega)\right\} .
\end{gathered}
$$


The spaces $L^{2}(\Omega)^{d}, H^{1}(\Omega)^{d}, \mathscr{H}$, and $\mathscr{H}_{1}$ are real Hilbert spaces endowed with the canonical inner products given by

$$
\begin{gathered}
(\mathbf{u}, \mathbf{v})_{L^{2}(\Omega)^{d}}=\int_{\Omega} \mathbf{u} \cdot \mathbf{v} d x, \quad(\mathbf{u}, \mathbf{v})_{H^{1}(\Omega)^{d}}=\int_{\Omega} \mathbf{u} \cdot \mathbf{v} d x+\int_{\Omega} \nabla \mathbf{u} \cdot \nabla \mathbf{v} d x, \\
(\boldsymbol{\sigma}, \boldsymbol{\tau})_{\mathscr{H}}=\int_{\Omega} \boldsymbol{\sigma} \cdot \boldsymbol{\tau} d x, \quad(\boldsymbol{\sigma}, \boldsymbol{\tau})_{\mathscr{H}_{1}}=\int_{\Omega} \boldsymbol{\sigma} \cdot \boldsymbol{\tau} d x+\int_{\Omega} \operatorname{Div} \boldsymbol{\sigma} \cdot \operatorname{Div} \boldsymbol{\tau} d x
\end{gathered}
$$

and the associated norms $\|\cdot\|_{L^{2}(\Omega)^{d}},\|\cdot\|_{H^{1}(\Omega)^{d}},\|\cdot\| \mathscr{H}$, and $\|\cdot\|_{\mathscr{K}_{1}}$, respectively. Here and below we use the notation

$$
\begin{aligned}
& \nabla \mathbf{v}=\left(v_{i, j}\right), \quad \boldsymbol{\varepsilon}(\mathbf{v})=\left(\varepsilon_{i j}(\mathbf{v})\right), \quad \varepsilon_{i j}(\mathbf{v})=\frac{1}{2}\left(v_{i, j}+v_{j, i}\right) \quad \forall \mathbf{v} \in H^{1}(\Omega)^{d}, \\
& \operatorname{Div} \boldsymbol{\tau}=\left(\tau_{i j, j}\right) \quad \forall \boldsymbol{\tau} \in \mathscr{H}_{1} .
\end{aligned}
$$

For every element $\mathbf{v} \in H^{1}(\Omega)^{d}$, we also write $\mathbf{v}$ for the trace of $\mathbf{v}$ on $\Gamma$ and we denote by $v_{v}$ and $\mathbf{v}_{\tau}$ the normal and tangential components of $\mathbf{v}$ on $\Gamma$ given by $v_{v}=\mathbf{v} \cdot \boldsymbol{v}, \mathbf{v}_{\tau}=$ $\mathbf{v}-v_{\nu} \boldsymbol{v}$.

Let us now consider the closed subspace of $H^{1}(\Omega)^{d}$ defined by

$$
V=\left\{\mathbf{v} \in H^{1}(\Omega)^{d} \mid \mathbf{v}=\mathbf{0} \text { on } \Gamma_{1}\right\}
$$

Since meas $\left(\Gamma_{1}\right)>0$, the following Korn's inequality holds:

$$
\|\boldsymbol{\varepsilon}(\mathbf{v})\|_{\mathscr{H}} \geq c_{K}\|\mathbf{v}\|_{H^{1}(\Omega)^{d}} \quad \forall \mathbf{v} \in V
$$

where $c_{K}>0$ is a constant which depends only on $\Omega$ and $\Gamma_{1}$. Over the space $V$ we consider the inner product given by

$$
(\mathbf{u}, \mathbf{v})_{V}=(\varepsilon(\mathbf{u}), \boldsymbol{\varepsilon}(\mathbf{v}))_{\mathscr{H}}
$$

and let $\|\cdot\|_{V}$ be the associated norm. It follows from Korn's inequality (3.6) that $\|\cdot\|_{H^{1}(\Omega)^{d}}$ and $\|\cdot\|_{V}$ are equivalent norms on $V$ and, therefore, $\left(V,\|\cdot\|_{V}\right)$ is a real Hilbert space. Moreover, by the Sobolev trace theorem, (3.6) and (3.7), there exists a constant $c_{0}$ depending only on the domain $\Omega, \Gamma_{1}$ and $\Gamma_{3}$ such that

$$
\|\mathbf{v}\|_{L^{2}\left(\Gamma_{3}\right)^{d}} \leq c_{0}\|\mathbf{v}\|_{V} \quad \forall \mathbf{v} \in V
$$

We also introduce the spaces

$$
\begin{gathered}
W=\left\{\psi \in H^{1}(\Omega) \mid \psi=0 \text { on } \Gamma_{a}\right\}, \\
\mathcal{W}_{1}=\left\{\mathbf{E}=\left(E_{i}\right) \mid E_{i} \in L^{2}(\Omega), E_{i, i} \in L^{2}(\Omega)\right\} .
\end{gathered}
$$


8 Electroelastic contact problems with adhesion

Since meas $\left(\Gamma_{a}\right)>0$, the following Friedrichs-Poincaré inequality holds:

$$
\|\nabla \psi\|_{L^{2}(\Omega)^{d}} \geq c_{F}\|\psi\|_{H^{1}(\Omega)} \quad \forall \psi \in W
$$

where $c_{F}>0$ is a constant which depends only on $\Omega$ and $\Gamma_{a}$ and $\nabla \psi=(\psi, i)$. Over the space $W$ we consider the inner product given by

$$
(\varphi, \psi)_{W}=\int_{\Omega} \nabla \varphi \cdot \nabla \psi d x
$$

and let $\|\cdot\|_{W}$ be the associated norm. It follows from (3.10) that $\|\cdot\|_{H^{1}(\Omega)}$ and $\|\cdot\|_{W}$ are equivalent norms on $W$ and, therefore, $\left(W,\|\cdot\|_{W}\right)$ is a real Hilbert space. Moreover, the space $W_{1}$ is real Hilbert spaces with the inner product

$$
(\mathbf{D}, \mathbf{E})_{\mathscr{W}_{1}}=\int_{\Omega} \mathbf{D} \cdot \mathbf{E} d x+\int_{\Omega} \operatorname{div} \mathbf{D} \cdot \operatorname{div} \mathbf{E} d x
$$

and the associated norm $\|\cdot\|_{\mathscr{W}_{1}}$. Here and below we denote $\operatorname{div} \mathbf{E}=\left(E_{i, i}\right)$ for all $\mathbf{E} \in \mathcal{W}_{1}$.

For every real Hilbert space $X$ we use the classical notation for the spaces $L^{p}(0, T ; X)$ and $W^{k, p}(0, T ; X), 1 \leq p \leq \infty, k=1,2, \ldots$; we also use the space of continuous functions on $[0, T]$ with values on $X$, denoted by $C([0, T] ; X)$, equipped with the norm

$$
\|x\|_{C([0, T] ; X)}=\max _{t \in[0, T]}\|x(t)\|_{X},
$$

and we introduce the set

$$
2=\left\{\theta \in L^{\infty}\left(0, T ; L^{2}\left(\Gamma_{3}\right)\right) \mid 0 \leq \theta(t) \leq 1 \forall t \in[0, T] \text {, a.e. on } \Gamma_{3}\right\} .
$$

Finally, if $X_{1}$ and $X_{2}$ are two Hilbert spaces endowed with the inner products $(\cdot, \cdot)_{X_{1}}$ and $(\cdot, \cdot)_{X_{2}}$ and the associated norms $\|\cdot\|_{X_{1}}$ and $\|\cdot\|_{X_{2}}$, respectively, we denote by $X_{1} \times X_{2}$ the product space together with the canonical inner product $(\cdot, \cdot)_{X_{1} \times X_{2}}$ and the associated norm $\|\cdot\|_{X_{1} \times X_{2}}$.

In the study of Problems 2.1 and 2.2 we assume that the elasticity operator $\mathscr{A}$, the piezoelectric operator $\mathscr{E}$, and the electric permittivity operator $\mathscr{B}$ satisfy the following:

$$
\begin{aligned}
& \mathscr{A}: \Omega \times \mathbb{R}^{d} \longrightarrow \mathbb{R}^{d}, \\
& \mathscr{A}(\mathbf{x}, \boldsymbol{\xi})=\left(a_{i j k l}(\mathbf{x}) \xi_{k l}\right) \quad \forall \boldsymbol{\xi}=\left(\xi_{i j}\right) \in \mathbb{S}^{d}, \text { a.e. } \mathbf{x} \in \Omega, \\
& a_{i j k l}=a_{k l i j}=a_{i j l k} \in L^{\infty}(\Omega), \\
& \exists m_{\mathscr{A}}>0 \text { such that } \\
& a_{i j k l}(\mathbf{x}) \xi_{i j} \xi_{k l} \geq m_{\mathscr{A}}\|\boldsymbol{\xi}\|^{2} \quad \forall \boldsymbol{\xi}=\left(\xi_{i j}\right) \in \mathbb{S}^{d} \text {, a.e. } \mathbf{x} \in \Omega, \\
& \mathscr{E}: \Omega \times \mathbb{S}^{d} \longrightarrow \mathbb{R}^{d}, \\
& \mathscr{E}(\mathbf{x}, \boldsymbol{\tau})=\left(e_{i j k}(\mathbf{x}) \tau_{j k}\right) \quad \forall \boldsymbol{\tau}=\left(\boldsymbol{\tau}_{i j}\right) \in \mathbb{S}^{d}, \text { a.e. } \mathbf{x} \in \Omega, \\
& e_{i j k}=e_{i k j} \in L^{\infty}(\Omega),
\end{aligned}
$$




$$
\begin{aligned}
& \mathscr{B}: \Omega \times \mathbb{R}^{d} \longrightarrow \mathbb{R}^{d}, \\
& \mathscr{B}(\mathbf{x}, \mathbf{E})=\left(b_{i j}(\mathbf{x}) E_{j}\right) \quad \forall \mathbf{E}=\left(E_{i}\right) \in \mathbb{R}^{d}, \text { a.e. } \mathbf{x} \in \Omega, \\
& b_{i j}=b_{j i} \in L^{\infty}(\Omega), \\
& \exists m_{\mathscr{B}}>0 \text { such that } \\
& b_{i j}(\mathbf{x}) E_{i} E_{j} \geq m_{\mathscr{B}}\|\mathbf{E}\|^{2} \quad \forall \mathbf{E}=\left(E_{i}\right) \in \mathbb{R}^{d}, \text { a.e. } \mathbf{x} \in \Omega .
\end{aligned}
$$

These assumptions show that the elasticity operator $\mathscr{A}$, the piezoelectric operator $\mathscr{E}$, and the electric permittivity operator $\mathscr{B}$ are linear have measurable bounded components denoted by $a_{i j k l}, e_{i j k}$, and $b_{i j}$, respectively, and, moreover, $\mathscr{A}$ and $\mathscr{B}$ are symmetric and positive definite. Recall also that the transposed operator $\mathscr{E} *$ is given by $\mathscr{E} *=\left(e_{i j k}^{*}\right)$, where $e_{i j k}^{*}=e_{k i j}$, and the following equality holds:

$$
\mathscr{E} \boldsymbol{\sigma} \cdot \mathbf{v}=\boldsymbol{\sigma} \cdot \mathscr{E}^{*} \mathbf{v} \quad \forall \boldsymbol{\sigma} \in \mathbb{S}^{d}, \mathbf{v} \in \mathbb{R}^{d}
$$

The normal compliance function $p_{\nu}$ and the tangential function $p_{\tau}$ satisfy the following:

$$
p_{\nu}: \Gamma_{3} \times \mathbb{R} \longrightarrow \mathbb{R}_{+},
$$

$\exists L_{v}>0$ such that

$$
\begin{aligned}
& \left|p_{\nu}\left(\mathbf{x}, r_{1}\right)-p_{\nu}\left(\mathbf{x}, r_{2}\right)\right| \leq L_{\nu}\left|r_{1}-r_{2}\right| \quad \forall r_{1}, r_{2} \in \mathbb{R}, \text { a.e. } \mathbf{x} \in \Gamma_{3}, \\
& \left(p_{\nu}\left(\mathbf{x}, r_{1}\right)-p_{\nu}\left(\mathbf{x}, r_{2}\right)\right)\left(r_{1}-r_{2}\right) \geq 0 \quad \forall r_{1}, r_{2} \in \mathbb{R}, \text { a.e. } \mathbf{x} \in \Gamma_{3},
\end{aligned}
$$

the mapping $\mathbf{x} \longmapsto p_{\nu}(\mathbf{x}, r)$ is measurable on $\Gamma_{3}, \quad$ for any $r \in \mathbb{R}$,

$p_{\nu}(\mathbf{x}, r)=0 \quad \forall r \leq 0$, a.e. $\mathbf{x} \in \Gamma_{3}$,

$p_{\tau}: \Gamma_{3} \times \mathbb{R} \longrightarrow \mathbb{R}_{+}$,

$\exists L_{\tau}>0$ such that

$\left|p_{\tau}\left(\mathbf{x}, \beta_{1}\right)-p_{\tau}\left(\mathbf{x}, \beta_{2}\right)\right| \leq L_{\tau}\left|\beta_{1}-\beta_{2}\right| \quad \forall \beta_{1}, \beta_{2} \in \mathbb{R}$, a.e. $\mathbf{x} \in \Gamma_{3}$,

$\exists M_{\tau}>0$ such that

$\left|p_{\tau}(\mathbf{x}, \beta)\right| \leq M_{\tau} \quad \forall \beta \in \mathbb{R}$, a.e. $\mathbf{x} \in \Gamma_{3}$,

the mapping $\mathbf{x} \longmapsto p_{\tau}(\mathbf{x}, \beta)$ is measurable on $\Gamma_{3}, \quad$ for any $\beta \in \mathbb{R}$,

the mapping $\mathbf{x} \longmapsto p_{\tau}(\mathbf{x}, 0)$ belongs to $L^{2}\left(\Gamma_{3}\right)$.

Various examples of functions $p_{\nu}$ and $p_{\tau}$ which satisfy conditions (3.19) and (3.20) can be found in $[20,23]$. Here we restrict ourselves to remark that the function $p_{\nu}(r)=r_{+}$ satisfies condition (3.19) and we conclude that our results below are valid for the corresponding contact problem. 
We also suppose that the body forces and surface tractions have the regularity

$$
\mathbf{f}_{0} \in W^{1, \infty}\left(0, T ; L^{2}(\Omega)^{d}\right), \quad \mathbf{f}_{2} \in W^{1, \infty}\left(0, T ; L^{2}\left(\Gamma_{2}\right)^{d}\right),
$$

and the densities of electric charges satisfy

$$
\begin{gathered}
q_{0} \in W^{1, \infty}\left(0, T ; L^{2}(\Omega)\right), \quad q_{2} \in W^{1, \infty}\left(0, T ; L^{2}\left(\Gamma_{b}\right)\right), \\
q_{2}(t)=0 \quad \text { on } \Gamma_{3} \forall t \in[0, T] .
\end{gathered}
$$

Note that we need to impose assumption (3.23) for physical reasons; indeed, the foundation is supposed to be insulator and therefore the electric boundary conditions on $\Gamma_{3}$ do not have to change in function of the status of the contact, are the same on the contact and on the separation zone, and are included in the boundary condition (2.11) or (2.27).

We define the functions $\mathbf{f}:[0, T] \rightarrow V$ and $q:[0, T] \rightarrow W$ by

$$
\begin{aligned}
& (\mathbf{f}(t), \mathbf{v})_{V}=\int_{\Omega} \mathbf{f}_{0}(t) \cdot \mathbf{v} d x+\int_{\Gamma_{2}} \mathbf{f}_{2}(t) \cdot \mathbf{v} d a, \\
& (q(t), \psi)_{W}=\int_{\Omega} q_{0}(t) \psi d x-\int_{\Gamma_{b}} q_{2}(t) \psi d a,
\end{aligned}
$$

for all $\mathbf{u}, \mathbf{v} \in V, \psi \in W$, and $t \in[0, T]$, and note that conditions (3.21) and (3.22) imply that

$$
\mathbf{f} \in W^{1, \infty}(0, T ; V), \quad q \in W^{1, \infty}(0, T ; W) .
$$

The adhesion coefficients $\gamma_{\nu}, \gamma_{\tau}$, and $\epsilon_{a}$ satisfy the conditions

$$
\gamma_{\nu}, \gamma_{\tau} \in L^{\infty}\left(\Gamma_{3}\right), \quad \epsilon_{a} \in L^{2}\left(\Gamma_{3}\right), \quad \gamma_{v}, \gamma_{\tau}, \epsilon_{a} \geq 0 \text { a.e. on } \Gamma_{3}
$$

and, finally, the initial data satisfies

$$
\beta_{0} \in L^{2}\left(\Gamma_{3}\right), \quad 0 \leq \beta_{0} \leq 1 \text { a.e. on } \Gamma_{3} .
$$

For the Signorini problem, we use the convex subset of admissible displacements given by

$$
U=\left\{\mathbf{v} \in V \mid v_{v} \leq 0 \text { on } \Gamma_{3}\right\}
$$

as well as the adhesion functional $j_{\text {ad }}: L^{\infty}\left(\Gamma_{3}\right) \times V \times V \rightarrow \mathbb{R}$ defined by

$$
j_{\mathrm{ad}}(\beta, \mathbf{u}, \mathbf{v})=\int_{\Gamma_{3}}\left(-\gamma_{\nu} \beta^{2} R_{\nu}\left(u_{\nu}\right) v_{\nu}+p_{\tau}(\beta) \mathbf{R}_{\tau}\left(\mathbf{u}_{\tau}\right) \cdot \mathbf{v}_{\tau}\right) d a .
$$

For the problem with normal compliance, in addition to the functional (3.29), we need the normal compliance functional $j_{\mathrm{nc}}: V \times V \rightarrow \mathbb{R}$ given by

$$
j_{\mathrm{nc}}(\mathbf{u}, \mathbf{v})=\int_{\Gamma_{3}} p_{\nu}\left(u_{\nu}\right) v_{\nu} d a
$$


By a standard procedure based on Green's formula we can derive the following variational formulation of the Signorini contact problem (2.1)-(2.12).

Problem 3.1. Find a displacement field $\mathbf{u}:[0, T] \rightarrow V$, an electric potential field $\varphi:[0$, $T] \rightarrow W$, and a bonding field $\beta:[0, T] \rightarrow L^{\infty}\left(\Gamma_{3}\right)$ such that

$$
\begin{gathered}
\mathbf{u}(t) \in U, \quad(\mathscr{A} \boldsymbol{\varepsilon}(\mathbf{u}(t)), \boldsymbol{\varepsilon}(\mathbf{v}-\mathbf{u}(t)))_{\mathscr{H}}+\left(\mathscr{E}^{*} \nabla \varphi(t), \boldsymbol{\varepsilon}(\mathbf{v}-\mathbf{u}(t))\right)_{\mathscr{H}}+j_{\mathrm{ad}}(\beta(t), \mathbf{u}(t), \mathbf{v}-\mathbf{u}(t)) \\
\geq(\mathbf{f}(t), \mathbf{v}-\mathbf{u}(t))_{V} \quad \forall \mathbf{v} \in U, t \in[0, T]
\end{gathered}
$$

$$
\begin{gathered}
(\mathscr{B} \nabla \varphi(t), \nabla \psi)_{L^{2}(\Omega)^{d}}-(\mathscr{E} \boldsymbol{\varepsilon}(\mathbf{u}(t)), \nabla \psi)_{L^{2}(\Omega)^{d}}=(q(t), \psi)_{W} \quad \forall \psi \in W, t \in[0, T], \\
\dot{\beta}(t)=-\left(\beta(t)\left(\gamma_{\nu} R_{\nu}\left(u_{\nu}(t)\right)^{2}+\gamma_{\tau}\left\|\mathbf{R}_{\tau}\left(\mathbf{u}_{\tau}(t)\right)\right\|^{2}\right)-\epsilon_{a}\right)_{+} \quad \text { a.e. } t \in(0, T), \\
\beta(0)=\beta_{0} .
\end{gathered}
$$

The variational formulation of the problem with normal compliance (2.17)-(2.28) is as follows.

Problem 3.2. Find a displacement field $\mathbf{u}_{\mu}:[0, T] \rightarrow V$, an electric potential field $\varphi_{\mu}$ : $[0, T] \rightarrow W$, and a bonding field $\beta_{\mu}:[0, T] \rightarrow L^{\infty}\left(\Gamma_{3}\right)$ such that

$$
\begin{gathered}
\left(\mathscr{A} \boldsymbol{\varepsilon}\left(\mathbf{u}_{\mu}(t)\right), \boldsymbol{\varepsilon}(\mathbf{v})\right)_{\mathscr{H}}+\left(\mathscr{E} * \nabla \varphi_{\mu}(t), \boldsymbol{\varepsilon}(\mathbf{v})\right)_{\mathscr{H}}+j_{\mathrm{ad}}\left(\beta_{\mu}(t), \mathbf{u}_{\mu}(t), \mathbf{v}\right)+\frac{1}{\mu} j_{\mathrm{nc}}\left(\mathbf{u}_{\mu}(t), \mathbf{v}\right) \\
=(\mathbf{f}(t), \mathbf{v})_{V} \quad \forall \mathbf{v} \in V, t \in[0, T], \\
\left(\mathscr{B} \nabla \varphi_{\mu}(t), \nabla \psi\right)_{L^{2}(\Omega)^{d}}-\left(\mathscr{E} \boldsymbol{\varepsilon}\left(\mathbf{u}_{\mu}(t)\right), \nabla \psi\right)_{L^{2}(\Omega)^{d}}=(q(t), \psi)_{W} \quad \forall \psi \in W, t \in[0, T], \\
\dot{\beta}_{\mu}(t)=-\left(\beta_{\mu}(t)\left(\gamma_{\nu} R_{\nu}\left(u_{\mu \nu}(t)\right)^{2}+\gamma_{\tau}\left\|\mathbf{R}_{\tau}\left(\mathbf{u}_{\mu \tau}(t)\right)\right\|^{2}\right)-\epsilon_{a}\right)_{+} \quad \text { a.e. } t \in(0, T), \\
\beta_{\mu}(0)=\beta_{0} .
\end{gathered}
$$

Remark 3.3. We note that the restrictions $0 \leq \beta \leq 1$ and $0 \leq \beta_{\mu} \leq 1$ are implicitly included in the variational Problems 3.1 and 3.2, respectively. Indeed, (3.33) and (3.34) guarantee that $\beta(\mathbf{x}, t) \leq \beta_{0}(\mathbf{x})$ and, therefore, assumption (3.27) shows that $\beta(\mathbf{x}, t) \leq 1$ for $t \geq 0$, a.e. $\mathbf{x} \in \Gamma_{3}$. On the other hand, if $\beta\left(\mathbf{x}, t_{0}\right)=0$ at time $t_{0}$, then it follows from (3.33) and (3.34) that $\dot{\beta}(\mathbf{x}, t)=0$ for all $t \geq t_{0}$ and, therefore, $\beta(\mathbf{x}, t)=0$ for all $t \geq t_{0}$, a.e. $\mathbf{x} \in \Gamma_{3}$. We conclude that $0 \leq \beta(\mathbf{x}, t) \leq 1$ for all $t \in[0, T]$, a.e. $\mathbf{x} \in \Gamma_{3}$. The same arguments show that $0 \leq \beta_{\mu}(\mathbf{x}, t) \leq 1$ for all $t \in[0, T]$, a.e. $\mathbf{x} \in \Gamma_{3}$.

Our main existence and uniqueness results which we state here and prove in the next section are the following. 
Theorem 3.4. Assume that (3.15)-(3.17), (3.20)-(3.23), and (3.26)-(3.27) hold. Then Problem 3.1 has a unique solution $(\mathbf{u}, \varphi, \beta)$. Moreover, the solution satisfies

$$
\begin{gathered}
\mathbf{u} \in W^{1, \infty}(0, T ; V), \\
\varphi \in W^{1, \infty}(0, T ; W), \\
\beta \in W^{1, \infty}\left(0, T ; L^{2}\left(\Gamma_{3}\right)\right) \cap 2 .
\end{gathered}
$$

Theorem 3.5. Assume that (3.15)-(3.17), (3.19)-(3.23), and (3.26)-(3.27) hold. Then, for all $\mu>0$, Problem 3.2 has a unique solution $\left(\mathbf{u}_{\mu}, \varphi_{\mu}, \beta_{\mu}\right)$ with the regularity expressed in (3.39)-(3.41).

A "quintuple" of functions $(\mathbf{u}, \boldsymbol{\sigma}, \varphi, \mathbf{D}, \beta)$ which satisfy (2.1), (2.2), and (3.31)-(3.34) is called a weak solution of the contact Problem 2.1. We conclude by Theorem 3.4 that, under the stated assumptions, Problem 2.1 has a unique weak solution. To precise the regularity of the weak solution we note that the constitutive relations (2.1) and (2.2), the assumptions (3.15)-(3.17), and the regularities (3.39), (3.40) show that $\sigma \in W^{1, \infty}(0, T ; \mathcal{H})$, $\mathbf{D} \in W^{1, \infty}\left(0, T ; L^{2}(\Omega)^{d}\right)$; moreover, (3.31), (3.32) combined with the definitions of $\mathbf{f}$ and $q$ yield

$$
\operatorname{Div} \boldsymbol{\sigma}(t)+\mathbf{f}_{0}(t)=\mathbf{0}, \quad \operatorname{div} \mathbf{D}(t)=q_{0}(t) \quad \forall t \in[0, T] .
$$

It follows now from the regularities (3.21), (3.22) that $\operatorname{Div} \sigma \in W^{1, \infty}\left(0, T ; L^{2}(\Omega)^{d}\right)$ and $\operatorname{div} \mathbf{D} \in W^{1, \infty}\left(0, T ; L^{2}(\Omega)\right)$, which shows that

$$
\begin{gathered}
\boldsymbol{\sigma} \in W^{1, \infty}\left(0, T ; \mathscr{H}_{1}\right), \\
\mathbf{D} \in W^{1, \infty}\left(0, T ; \mathcal{W}_{1}\right) .
\end{gathered}
$$

We conclude that the weak solution $(\mathbf{u}, \boldsymbol{\sigma}, \varphi, \mathbf{D}, \beta)$ of the piezoelectric contact Problem 2.1 has the regularity (3.39)-(3.44).

A "quintuple" of functions $\left(\mathbf{u}_{\mu}, \boldsymbol{\sigma}_{\mu}, \varphi_{\mu}, \mathbf{D}_{\mu}, \beta_{\mu}\right)$ which satisfy (2.17), (2.18), and (3.35)(3.38) is called a weak solution of the electroelastic contact Problem 2.2. We conclude by Theorem 3.5 and the arguments above that, under the stated assumptions, for all $\mu>0$ Problem 2.2 has a unique weak solution, and it has the regularity expressed in (3.39)(3.44).

The behavior of the solution $\left(\mathbf{u}_{\mu}, \varphi_{\mu}, \beta_{\mu}\right)$ as $\mu \rightarrow 0$ is given by the following result that we state here and prove in Section 5.

Theorem 3.6. Assume that (3.15)-(3.17), (3.19)-(3.23), and (3.26)-(3.27) hold and assume moreover that

$$
p_{\nu}(\mathbf{x}, r)=0 \quad \text { iff } r \leq 0 \text {, a.e. } \mathbf{x} \in \Gamma_{3} \text {. }
$$


Then the solution $\left(\mathbf{u}_{\mu}, \varphi_{\mu}, \beta_{\mu}\right)$ of problem (3.35)-(3.38) converges to the solution $(\mathbf{u}, \varphi, \beta)$ of problem (3.31)-(3.34), that is,

$$
\left\|\mathbf{u}_{\mu}(t)-\mathbf{u}(t)\right\|_{V}+\left\|\varphi_{\mu}(t)-\varphi(t)\right\|_{W}+\left\|\beta_{\mu}(t)-\beta(t)\right\|_{L^{2}\left(\Gamma_{3}\right)} \longrightarrow 0
$$

as $\mu \rightarrow 0$, for all $t \in[0, T]$.

In addition to the mathematical interest in the result above, it is important from the physical point of view, since it shows that the weak solution of the electroelastic adhesive contact problem with a rigid obstacle may be approached as closely as one wishes by the solution of the electroelastic adhesive contact problem with a deformable foundation, with a sufficiently small deformability coefficient. Theorem 3.6 is also important from numerical point of view since it justifies the use of the normal compliance contact condition as an approximation of the Signorini condition, in the numerical treatment of Problem 2.1.

In the proofs of Theorems 3.4-3.6 we use a number of inequalities involving the functionals $j_{\mathrm{ad}}$ and $j_{\mathrm{nc}}$ that we present in what follows. Below in this section $\beta, \beta_{1}, \beta_{2}$ denote elements of $L^{2}\left(\Gamma_{3}\right)$ such that $0 \leq \beta, \beta_{1}, \beta_{2} \leq 1$ a.e. on $\Gamma_{3}, \mathbf{u}_{1}, \mathbf{u}_{2}$, and $\mathbf{v}$ represent elements of $V$ and $c>0$ represent generic constants which may depend on $\Omega, \Gamma_{1}, \Gamma_{3}, p_{v}, p_{\tau}, \gamma_{\nu}, \gamma_{\tau}$, and $L$.

First, we notice that the $j_{\mathrm{ad}}$ and $j_{\mathrm{nc}}$ are linear with respect to the last argument and therefore

$$
j_{\mathrm{ad}}(\beta, \mathbf{u},-\mathbf{v})=-j_{\mathrm{ad}}(\beta, \mathbf{u}, \mathbf{v}), \quad j_{\mathrm{nc}}(\mathbf{u},-\mathbf{v})=-j_{\mathrm{nc}}(\mathbf{u}, \mathbf{v}) .
$$

Next, using (3.29), the properties of the truncation operators $R_{v}$ and $\mathbf{R}_{\tau}$ as well as assumption (3.20) on the function $p_{\tau}$, after some calculus, we find

$$
j_{\mathrm{ad}}\left(\beta_{1}, \mathbf{u}_{1}, \mathbf{u}_{2}-\mathbf{u}_{1}\right)+j_{\mathrm{ad}}\left(\beta_{2}, \mathbf{u}_{2}, \mathbf{u}_{1}-\mathbf{u}_{2}\right) \leq c \int_{\Gamma_{3}}\left|\beta_{1}-\beta_{2}\right||| \mathbf{u}_{1}-\mathbf{u}_{2}|| d a
$$

and, by (3.25), we obtain

$$
j_{\text {ad }}\left(\beta_{1}, \mathbf{u}_{1}, \mathbf{u}_{2}-\mathbf{u}_{1}\right)+j_{\text {ad }}\left(\beta_{2}, \mathbf{u}_{2}, \mathbf{u}_{1}-\mathbf{u}_{2}\right) \leq c\left\|\beta_{1}-\beta_{2}\right\|_{L^{2}\left(\Gamma_{3}\right)}\left\|\mathbf{u}_{1}-\mathbf{u}_{2}\right\|_{V} .
$$

Similar computations, based on the Lipschitz continuity of $R_{v}, \mathbf{R}_{\tau}$, and $p_{\tau}$, show that the following inequality also holds:

$$
\left|j_{\mathrm{ad}}\left(\beta, \mathbf{u}_{1}, \mathbf{v}\right)-j_{\mathrm{ad}}\left(\beta, \mathbf{u}_{2}, \mathbf{v}\right)\right| \leq c\left\|\mathbf{u}_{1}-\mathbf{u}_{2}\right\|_{V}\|\mathbf{v}\|_{V} .
$$

We now take $\beta_{1}=\beta_{2}=\beta$ in (3.49) to deduce

$$
j_{\mathrm{ad}}\left(\beta, \mathbf{u}_{1}, \mathbf{u}_{2}-\mathbf{u}_{1}\right)+j_{\mathrm{ad}}\left(\beta, \mathbf{u}_{2}, \mathbf{u}_{1}-\mathbf{u}_{2}\right) \leq 0 .
$$

Also, we take $\mathbf{u}_{1}=\mathbf{v}$ and $\mathbf{u}_{2}=\mathbf{0}$ in (3.51), then we use the equalities $R_{\nu}(0)=0, \mathbf{R}_{\tau}(\mathbf{0})=\mathbf{0}$, and (3.47) to obtain

$$
j_{\text {ad }}(\beta, \mathbf{v}, \mathbf{v}) \geq 0 .
$$


14 Electroelastic contact problems with adhesion

Now, we use (3.30) to see that

$$
\left|j_{\mathrm{nc}}\left(\mathbf{u}_{1}, \mathbf{v}\right)-j_{\mathrm{nc}}\left(\mathbf{u}_{2}, \mathbf{v}\right)\right| \leq \int_{\Gamma_{3}}\left|p_{\nu}\left(u_{1 \nu}\right)-p_{\nu}\left(u_{2 \nu}\right)\right|\left|v_{\nu}\right| d a
$$

and therefore (3.19b) and (3.8) imply

$$
\left|j_{\mathrm{nc}}\left(\mathbf{u}_{1}, \mathbf{v}\right)-j_{\mathrm{nc}}\left(\mathbf{u}_{2}, \mathbf{v}\right)\right| \leq c\left\|\mathbf{u}_{1}-\mathbf{u}_{2}\right\|_{V}\|\mathbf{v}\|_{V}
$$

We use again (3.30) to find

$$
j_{\mathrm{nc}}\left(\mathbf{u}_{1}, \mathbf{u}_{2}-\mathbf{u}_{1}\right)+j_{\mathrm{nc}}\left(\mathbf{u}_{2}, \mathbf{u}_{1}-\mathbf{u}_{2}\right)=\int_{\Gamma_{3}}\left(p_{\nu}\left(u_{1 v}\right)-p_{\nu}\left(u_{2 \gamma}\right)\right)\left(u_{2 \gamma}-u_{1 \nu}\right) d a
$$

and therefore $(3.19 \mathrm{c})$ implies

$$
j_{\mathrm{nc}}\left(\mathbf{u}_{1}, \mathbf{u}_{2}-\mathbf{u}_{1}\right)+j_{\mathrm{nc}}\left(\mathbf{u}_{2}, \mathbf{u}_{1}-\mathbf{u}_{2}\right) \leq 0
$$

Take $\mathbf{u}_{1}=\mathbf{v}$ and $\mathbf{u}_{2}=\mathbf{0}$ in the previous inequality and use (3.19e) and (3.47) to obtain

$$
j_{\mathrm{nc}}(\mathbf{v}, \mathbf{v}) \geq 0 \text {. }
$$

The inequalities (3.49)-(3.57) combined with equalities (3.47) will be used in various places in the rest of the paper.

\section{Proof of Theorems 3.4 and 3.5}

We turn now to the proof of Theorem 3.4 which will be carried out in several steps. To this end, we assume in the following that (3.15)-(3.17), (3.20)-(3.23), and (3.26)-(3.27) hold; below, $c$ denotes a generic positive constant which may depend on $\Omega, \Gamma_{1}, \Gamma_{3}, \mathscr{A}, \mathscr{B}$, $\mathscr{E}, \gamma_{v}, \gamma_{\tau}$, and $T$ but does not depend on $t$ nor on the rest of the input data, and whose value may change from place to place. Moreover, for the sake of simplicity, we suppress, in what follows, the explicit dependence of various functions on $\mathbf{x} \in \Omega \cup \Gamma$.

Let $\mathscr{Z}$ denote the closed set of the space $C\left([0, T] ; L^{2}\left(\Gamma_{3}\right)\right)$ defined by

$$
\mathscr{L}=\left\{\beta \in C\left([0, T] ; L^{2}\left(\Gamma_{3}\right)\right) \cap \mathscr{Q} \mid \beta(0)=\beta_{0}\right\}
$$

and let $\beta \in \mathscr{L}$ be given. In the first step we consider the following variational problem.

Problem 4.1. Find a displacement field $\mathbf{u}_{\beta}:[0, T] \rightarrow V$ and an electric potential $\varphi_{\beta}:[0, T]$ $\rightarrow W$ such that, for all $t \in[0, T]$,

$$
\begin{gathered}
\mathbf{u}_{\beta}(t) \in U, \quad\left(\mathscr{A} \boldsymbol{\varepsilon}\left(\mathbf{u}_{\beta}(t)\right), \boldsymbol{\varepsilon}\left(\mathbf{v}-\mathbf{u}_{\beta}(t)\right)\right)_{\mathscr{H}}+\left(\mathscr{E}^{*} \nabla \varphi_{\beta}(t), \boldsymbol{\varepsilon}\left(\mathbf{v}-\mathbf{u}_{\beta}(t)\right)\right)_{\mathscr{H}} \\
\quad+j_{\mathrm{ad}}\left(\beta(t), \mathbf{u}_{\beta}(t), \mathbf{v}-\mathbf{u}_{\beta}(t)\right) \\
\geq\left(\mathbf{f}(t), \mathbf{v}-\mathbf{u}_{\beta}(t)\right)_{V} \quad \forall \mathbf{v} \in U, \\
\left(\mathscr{B} \nabla \varphi_{\beta}(t), \nabla \psi\right)_{L^{2}(\Omega)^{d}}-\left(\mathscr{E} \boldsymbol{\varepsilon}\left(\mathbf{u}_{\beta}(t)\right), \nabla \psi\right)_{L^{2}(\Omega)^{d}}=(q(t), \psi)_{W} \quad \forall \psi \in W .
\end{gathered}
$$


In order to solve Problem 4.1 we consider the product space $X=V \times W$ endowed with the inner product

$$
(x, y)_{X}=(\mathbf{u}, \mathbf{v})_{V}+(\varphi, \psi)_{W} \quad \forall x=(\mathbf{u}, \varphi), y=(\mathbf{v}, \psi) \in X
$$

and the associated norm $\|\cdot\|_{X}$. We also introduce the set $K \subset X$ and the functions $A_{\beta}$ : $[0, T] \times X \rightarrow X, f:[0, T] \rightarrow X$, defined by

$$
\begin{gathered}
K=U \times W \\
\left(A_{\beta}(t) x, y\right)_{X}=(\mathscr{A} \boldsymbol{\varepsilon}(\mathbf{u}), \boldsymbol{\varepsilon}(\mathbf{v}))_{\mathscr{H}}+(\mathscr{S} \nabla \varphi, \nabla \psi)_{L^{2}(\Omega)^{d}}+(\mathscr{E} * \nabla \varphi, \boldsymbol{\varepsilon}(\mathbf{v}))_{\mathscr{H}}-(\mathscr{E} \boldsymbol{\varepsilon}(\mathbf{u}), \nabla \psi)_{L^{2}(\Omega)^{d}} \\
+j_{\mathrm{ad}}(\beta(t) \mathbf{u}, \mathbf{v}) \quad \forall x=(\mathbf{u}, \varphi), y=(\mathbf{v}, \psi) \in X, t \in[0, T] \\
f(t)=(\mathbf{f}(t), q(t)) \quad \forall t \in[0, T] .
\end{gathered}
$$

We start with the following equivalence result.

Lemma 4.2. The couple $x_{\beta}=\left(\mathbf{u}_{\beta}, \varphi_{\beta}\right):[0, T] \rightarrow V \times W$ is a solution to Problem 4.1 if and only if $x_{\beta}:[0, T] \rightarrow X$ satisfies

$$
x_{\beta}(t) \in K, \quad\left(A_{\beta}(t) x_{\beta}(t), y-x_{\beta}(t)\right) \geq\left(f(t), y-x_{\beta}(t)\right)_{X} \quad \forall y \in K,
$$

for all $t \in[0, T]$.

Proof. Let $x_{\beta}=\left(\mathbf{u}_{\beta}, \varphi_{\beta}\right):[0, T] \rightarrow V \times W$ be a solution to Problem 4.1. Let $y=(\mathbf{v}, \psi) \in K$ and let $t \in[0, T]$. We use the test function $\psi-\varphi_{\beta}(t)$ in (4.3), add the corresponding inequality to (4.2), and use (4.4)-(4.7) to obtain (4.8). Conversely, assume that $x_{\beta}=$ $\left(\mathbf{u}_{\beta}, \varphi_{\beta}\right):[0, T] \rightarrow X$ satisfies $(4.8)$ and let $t \in[0, T]$. For any $\mathbf{v} \in U$, we take $y=\left(\mathbf{v}, \varphi_{\beta}(t)\right)$ in (4.8) to obtain (4.2). Then, for any $\psi \in W$, we take successively $y=\left(\mathbf{u}_{\beta}, \varphi_{\beta}(t)+\psi\right)$ and $y=\left(\mathbf{u}_{\beta}, \varphi_{\beta}(t)-\psi\right)$ in (4.8) to obtain (4.3).

We use now Lemma 4.2 to obtain the following existence and uniqueness result.

Lemma 4.3. Problem 4.1 has a unique solution $\left(\mathbf{u}_{\beta}, \varphi_{\beta}\right) \in C([0, T] ; V \times W)$.

Proof. Let $t \in[0, T]$. We use (3.15)-(3.18), (3.50), and (3.51) to see that $A_{\beta}(t)$ is a strongly monotone Lipschitz continuous operator on $X$; since $K$ is a nonempty closed convex set of $X$, by a standard result on elliptic variational inequalities it follows that there exists a unique element $x_{\beta}(t)=\left(\mathbf{u}_{\beta}(t), \varphi_{\beta}(t)\right) \in X$ which satisfies (4.8). For $t_{1}, t_{2} \in[0, T]$, an argument based on (3.15) and (3.49) shows that

$$
\left\|x_{\beta}\left(t_{1}\right)-x_{\beta}\left(t_{2}\right)\right\|_{X} \leq c\left(\left\|\beta\left(t_{1}\right)-\beta\left(t_{2}\right)\right\|_{L^{2}\left(\Gamma_{3}\right)}+\left\|f\left(t_{1}\right)-f\left(t_{2}\right)\right\|_{X}\right) .
$$

By (3.25) and (4.7) we deduce that $f \in W^{1, \infty}(0, T ; X)$ and recall that $\beta \in C([0, T] ; X)$, see (4.1). It follows now from (4.9) that the mapping $t \mapsto x_{\beta}:[0, T] \rightarrow X$ is continuous. The existence and uniqueness part in Lemma 4.3 is now a consequence of Lemma 4.2. 
In the second step we use the displacement field $\mathbf{u}_{\beta}$ obtained in Lemma 4.3, denote by $u_{\beta \gamma}, \mathbf{u}_{\beta \tau}$ its normal and tangential components, and we consider the following initialvalue problem.

Problem 4.4. Find a bonding field $\theta_{\beta}:[0, T] \rightarrow L^{2}\left(\Gamma_{3}\right)$ such that

$$
\begin{gathered}
\dot{\theta}_{\beta}(t)=-\left(\theta_{\beta}(t)\left(\gamma_{\nu} R_{\nu}\left(u_{\beta \nu}(t)\right)^{2}+\gamma_{\tau}\left\|\mathbf{R}_{\tau}\left(\mathbf{u}_{\beta \tau}(t)\right)\right\|^{2}\right)-\epsilon_{a}\right)_{+} \text {a.e. } t \in(0, T), \\
\theta_{\beta}(0)=\beta_{0} .
\end{gathered}
$$

We obtain the following result.

Lemma 4.5. There exists a unique solution to Problem 4.4 and it satisfies $\theta_{\beta} \in W^{1, \infty}(0, T$, $\left.L^{2}\left(\Gamma_{3}\right)\right) \cap 2$.

Proof. Consider the mapping $F_{\beta}:[0, T] \times L^{2}\left(\Gamma_{3}\right) \rightarrow L^{2}\left(\Gamma_{3}\right)$ defined by

$$
F_{\beta}(t, \theta)=-\left(\gamma_{\nu} \theta\left(R_{\nu}\left(u_{\beta \nu}(t)\right)^{2}+\gamma_{\tau}\left\|\mathbf{R}_{\tau}\left(\mathbf{u}_{\beta \tau}(t)\right)\right\|^{2}\right)-\epsilon_{a}\right)_{+},
$$

for all $t \in[0, T]$ and $\theta \in L^{2}\left(\Gamma_{3}\right)$. It follows from the properties of the truncation operators $R_{v}$ and $\mathbf{R}_{\tau}$ that $F_{\beta}$ is Lipschitz continuous with respect to the second argument, uniformly in time. Moreover, for any $\theta \in L^{2}\left(\Gamma_{3}\right)$, the mapping $t \mapsto F_{\beta}(t, \theta)$ belongs to $L^{\infty}\left(0, T ; L^{2}\left(\Gamma_{3}\right)\right)$. Using now a version of Cauchy-Lipschitz theorem (see, e.g., [23, page $48])$, we obtain the existence of a unique function $\theta_{\beta} \in W^{1, \infty}\left(0, T, L^{2}\left(\Gamma_{3}\right)\right)$ which solves (4.10). Also, the arguments used in Remark 3.3 show that $0 \leq \theta_{\beta}(t) \leq 1$ for all $t \in[0, T]$, a.e. on $\Gamma_{3}$. Therefore, from the definition of the set 2 , we find that $\theta_{\beta} \in 2$, which concludes the proof of the lemma.

It follows from Lemma 4.5 that for all $\beta \in \mathscr{E}$ the solution $\theta_{\beta}$ of Problem 4.4 belongs to $\mathscr{L}$, see (4.1). Therefore, we may consider the operator $\Lambda: \mathscr{L} \rightarrow \mathscr{L}$ given by

$$
\Lambda \beta=\theta_{\beta}
$$

The third step consists in the following result.

Lemma 4.6. There exists a unique element $\beta^{*} \in \mathscr{L}$ such that $\Lambda \beta^{*}=\beta^{*}$.

Proof. Suppose that $\beta_{i}$ are two functions in $\mathscr{L}$ and denote by $\left(\mathbf{u}_{i}, \varphi_{i}\right), \theta_{i}$ the functions obtained in Lemmas 4.3 and 4.5, respectively, for $\beta=\beta_{i}, i=1,2$. Let $t \in[0, T]$. We use arguments similar to those used in the proof of (4.9) to deduce that

$$
\left\|\mathbf{u}_{1}(t)-\mathbf{u}_{2}(t)\right\|_{V}+\left\|\varphi_{1}(t)-\varphi_{2}(t)\right\|_{W} \leq c\left\|\beta_{1}(t)-\beta_{2}(t)\right\|_{L^{2}\left(\Gamma_{3}\right)}
$$

which implies that

$$
\left\|\mathbf{u}_{1}(t)-\mathbf{u}_{2}(t)\right\|_{V} \leq c\left\|\beta_{1}(t)-\beta_{2}(t)\right\|_{L^{2}\left(\Gamma_{3}\right)} .
$$

On the other hand, it follows from (4.10) that

$$
\theta_{i}(t)=\beta_{0}-\int_{0}^{t}\left(\theta_{i}(s)\left(\gamma_{\nu} R_{\nu}\left(u_{i v}(s)\right)^{2}+\gamma_{\tau}\left\|\mathbf{R}_{\tau}\left(\mathbf{u}_{i \tau}(s)\right)\right\|^{2}\right)-\epsilon_{a}\right)_{+} d s
$$


and then

$$
\begin{aligned}
\left.\left\|\theta_{1}(t)-\theta_{2}(t)\right\|\right|_{L^{2}\left(\Gamma_{3}\right)} \leq & c \int_{0}^{t}\left\|\theta_{1}(s) R_{\nu}\left(u_{1 \nu}(s)\right)^{2}-\theta_{2}(s) R_{\nu}\left(u_{2 \nu}(s)\right)^{2}\right\|_{L^{2}\left(\Gamma_{3}\right)} d s \\
& +\int_{0}^{t}\left\|\theta_{1}(s)\right\| \mathbf{R}_{\tau}\left(\mathbf{u}_{1 \tau}(s)\right)\left\|^{2}-\theta_{2}(s)\right\| \mathbf{R}_{\tau}\left(\mathbf{u}_{2 \tau}(s)\right)\left\|^{2}\right\|_{L^{2}\left(\Gamma_{3}\right)} d s .
\end{aligned}
$$

Using the definition of $R_{v}$ and $\mathbf{R}_{\tau}$ and writing $\theta_{1}=\theta_{1}-\theta_{2}+\theta_{2}$, we get

$$
\left\|\theta_{1}(t)-\theta_{2}(t)\right\|_{L^{2}\left(\Gamma_{3}\right)} \leq c \int_{0}^{t}\left\|\theta_{1}(s)-\theta_{2}(s)\right\|_{L^{2}\left(\Gamma_{3}\right)} d s+c \int_{0}^{t}\left\|\mathbf{u}_{1}(s)-\mathbf{u}_{2}(s)\right\|_{L^{2}\left(\Gamma_{3}\right)^{d}} d s .
$$

By Gronwall's inequality, it follows that

$$
\left\|\theta_{1}(t)-\theta_{2}(t)\right\|_{L^{2}\left(\Gamma_{3}\right)} \leq c \int_{0}^{t}\left\|\mathbf{u}_{1}(s)-\mathbf{u}_{2}(s)\right\|_{L^{2}\left(\Gamma_{3}\right)^{d}} d s
$$

and, using (3.8), we obtain

$$
\left\|\theta_{1}(t)-\theta_{2}(t)\right\|_{L^{2}\left(\Gamma_{3}\right)} \leq c \int_{0}^{t}\left\|\mathbf{u}_{1}(s)-\mathbf{u}_{2}(s)\right\|_{V} d s
$$

We use (4.12) and the estimate (4.19) to find

$$
\left\|\Lambda \beta_{1}(t)-\Lambda \beta_{2}(t)\right\|_{L^{2}\left(\Gamma_{3}\right)} \leq c \int_{0}^{t}\left\|\mathbf{u}_{1}(s)-\mathbf{u}_{2}(s)\right\|_{V} d s .
$$

We now combine (4.14) and (4.20) to see that

$$
\left\|\Lambda \beta_{1}(t)-\Lambda \beta_{2}(t)\right\|_{L^{2}\left(\Gamma_{3}\right)} \leq c \int_{0}^{t}\left\|\beta_{1}(s)-\beta_{2}(s)\right\|_{L^{2}\left(\Gamma_{3}\right)} d s
$$

and reiterating this inequality $m$ times yields

$$
\left\|\Lambda^{m} \beta_{1}-\Lambda^{m} \beta_{2}\right\|_{C\left([0, T] ; L^{2}\left(\Gamma_{3}\right)\right)} \leq \frac{c^{m} T^{m}}{m !}\left\|\beta_{1}-\beta_{2}\right\|_{C\left([0, T] ; L^{2}\left(\Gamma_{3}\right)\right)} .
$$

Recall that $\mathscr{L}$ is a nonempty closed set in the Banach space $C\left([0, T] ; L^{2}\left(\Gamma_{3}\right)\right)$ and note that inequality (4.22) shows that for $m$ sufficiently large $\Lambda^{m}: \mathscr{L} \rightarrow \mathscr{L}$ is a contraction. Then, by using the Banach fixed point theorem, it follows that $\Lambda$ has a unique fixed point $\beta^{*} \in \mathscr{L}$.

Now, we have all the ingredients to provide the proof of Theorem 3.4.

\section{Proof}

Existence. Let $\beta^{*} \in \mathscr{E}$ be the fixed point of $\Lambda$ and let $\left(\mathbf{u}^{*}, \varphi^{*}\right)$ be the solution of Problem 4.1 for $\beta=\beta^{*}$, that is, $\mathbf{u}^{*}=\mathbf{u}_{\beta^{*}}$ and $\varphi^{*}=\varphi_{\beta^{*}}$. Since $\theta_{\beta^{*}}=\beta^{*}$, we conclude by (4.2), (4.3), and (4.10) that $\left(\mathbf{u}^{*}, \varphi^{*}, \beta^{*}\right)$ is a solution of Problem 3.1 and, moreover, $\beta^{*}$ satisfies (3.41). Also, since $\beta^{*}=\theta_{\beta^{*}} \in W^{1, \infty}\left(0, T ; L^{2}\left(\Gamma_{3}\right)\right)$ and $f \in W^{1, \infty}(0, T ; X)$, inequality (4.9) 
implies that the function $x^{*}=\left(\mathbf{u}^{*}, \varphi^{*}\right):[0, T] \rightarrow X$ is Lipschitz continuous; therefore, $x^{*}$ belongs to $W^{1, \infty}(0, T ; X)$, which shows that the functions $\mathbf{u}^{*}$ and $\varphi^{*}$ have the regularity expressed in (3.39), (3.40).

Uniqueness. The uniqueness of the solution is a consequence of the uniqueness of the fixed point of operator $\Lambda$ defined by (4.12). Indeed, let $(\mathbf{u}, \varphi, \beta)$ be a solution of Problem 3.1 which satisfies (3.39)-(3.41). It follows from (4.2), (4.3) that $\mathbf{u}$ is a solution to Problem 4.1 and, since by Lemma 4.3 this problem has a unique solution denoted by $\left(\mathbf{u}_{\beta}, \varphi_{\beta}\right)$, we obtain

$$
\begin{aligned}
& \mathbf{u}=\mathbf{u}_{\beta}, \\
& \varphi=\varphi_{\beta} .
\end{aligned}
$$

Then, we replace $\mathbf{u}=\mathbf{u}_{\beta}$ in (3.33) and use the initial condition (3.34) to see that $\beta$ is a solution to Problem 4.4. Since by Lemma 4.5 this last problem has a unique solution denoted by $\theta_{\beta}$, we find

$$
\beta=\theta_{\beta}
$$

We use now (4.12) and (4.24) to see that $\Lambda \beta=\beta$, that is, $\beta$ is a fixed point of the operator $\Lambda$. It follows now from Lemma 4.6 that

$$
\beta=\beta^{*}
$$

The uniqueness part of the theorem is now a consequence of (4.23) and (4.25).

We turn now to the proof of Theorem 3.5 which is similar to the proof of Theorem 3.4 and it is carried out in several steps. Since the modifications are straightforward, we do not indicate the details. Below in this section we assume that $\mu>0$ is fixed and, for simplicity, we do not indicate the dependence of the solution on $\mu$.

Proof. (i) For any $\beta \in \mathscr{L}$, we prove that there exists a unique couple of functions $\left(\mathbf{u}_{\beta}, \varphi_{\beta}\right) \in$ $C([0, T] ; V \times W)$ such that, for all $t \in[0, T]$,

$$
\begin{aligned}
& \left(\mathscr{A} \boldsymbol{\varepsilon}\left(\mathbf{u}_{\beta}(t)\right), \boldsymbol{\varepsilon}(\mathbf{v})\right)_{\mathscr{H}}+\left(\mathscr{E}^{*} \nabla \varphi_{\beta}(t), \boldsymbol{\varepsilon}(\mathbf{v})\right)_{\mathscr{H}}+j_{\mathrm{ad}}\left(\beta(t), \mathbf{u}_{\beta}(t), \mathbf{v}\right)+\frac{1}{\mu} j_{\mathrm{nc}}\left(\mathbf{u}_{\beta}(t), \mathbf{v}\right) \\
& \quad=(\mathbf{f}(t), \mathbf{v})_{V} \quad \forall \mathbf{v} \in V, \\
& \left(\mathscr{B} \nabla \varphi_{\beta}(t), \nabla \psi\right)_{L^{2}(\Omega)^{d}}-\left(\mathscr{E} \boldsymbol{\varepsilon}\left(\mathbf{u}_{\beta}(t)\right), \nabla \psi\right)_{L^{2}(\Omega)^{d}}=(q(t), \psi)_{W} \quad \forall \psi \in W .
\end{aligned}
$$

To provide this step we use arguments similar to those used in the proof of Lemmas 4.2 and 4.3. The main difference arises from the fact that now (4.8) is replaced by the equality

$$
x_{\beta}(t) \in X, \quad\left(A_{\beta}(t) x_{\beta}(t), y\right)_{X}=(f(t), y)_{X} \quad \forall y \in X, t \in[0, T]
$$


where, for $\beta \in \mathscr{L}$ and $t \in[0, T], A_{\beta}(t): X \rightarrow X$ is the operator defined by

$$
\begin{aligned}
\left(A_{\beta}(t) x, y\right)_{X}= & (\mathscr{A} \boldsymbol{\varepsilon}(\mathbf{u}), \boldsymbol{\varepsilon}(\mathbf{v}))_{\mathscr{H}}+(\mathscr{B} \nabla \varphi, \nabla \psi)_{L^{2}(\Omega)^{d}}+(\mathscr{E} * \nabla \varphi, \boldsymbol{\varepsilon}(\mathbf{v}))_{\mathscr{H}}-(\mathscr{E} \boldsymbol{\varepsilon}(\mathbf{u}), \nabla \psi)_{L^{2}(\Omega)^{d}} \\
& +j_{\mathrm{ad}}(\beta(t) \mathbf{u}, \mathbf{v})+\frac{1}{\mu} j_{\mathrm{nc}}(\mathbf{u}, \mathbf{v}) \quad \forall x=(\mathbf{u}, \varphi), y=(\mathbf{v}, \psi) \in X
\end{aligned}
$$

We use (3.15)-(3.18) and the properties (3.47)-(3.57) of the functionals $j_{\text {ad }}$ and $j_{\text {nc }}$ to see that $A_{\beta}(t)$ is again a strongly monotone Lipschitz continuous operator.

(ii) For a given $\beta \in \mathscr{L}$, we prove that there exists a unique element $\theta$ such that

$$
\begin{gathered}
\theta_{\beta} \in W^{1, \infty}\left(0, T ; L^{2}\left(\Gamma_{3}\right)\right) \cap \mathscr{Z}, \\
\dot{\theta}_{\beta}(t)=-\left(\gamma_{\nu} \theta_{\beta}(t)\left(R_{\nu}\left(u_{\beta \nu}(t)\right)^{2}+\gamma_{\tau}\left\|\mathbf{R}_{\tau}\left(\mathbf{u}_{\beta \tau}(t)\right)\right\|^{2}\right)-\epsilon_{a}\right)_{+} \quad \text { a.e. } t \in(0, T), \\
\theta_{\beta}(0)=\beta_{0} .
\end{gathered}
$$

The proof of this step is based on Lemma 4.5.

(iii) The operator $\Lambda: \mathscr{L} \rightarrow \mathscr{L}$ given by

$$
\Lambda \beta=\theta_{\beta}
$$

has a unique element $\beta^{*} \in \mathscr{L}$. The proof is based on estimates similar to those presented in Lemma 4.6 and the Banach fixed point theorem.

(iv) Let $\beta^{*} \in \mathscr{L}$ be the fixed point of $\Lambda$ and denote $\mathbf{u}^{*}=\mathbf{u}_{\beta^{*}}, \varphi^{*}=\varphi_{\beta^{*}}$, where $\left(\mathbf{u}_{\beta^{*}}\right.$, $\varphi_{\beta^{*}}$ ) is the couple of functions obtained in step (i) for $\beta=\beta^{*}$. Then, we use (4.26)(4.30) to see that $\left(\mathbf{u}^{*}, \varphi^{*}, \beta^{*}\right)$ is the unique solution of Problem 3.2 and it satisfies (3.39)(3.41).

\section{Proof of Theorem 3.6}

The proof of Theorem 3.6 is carried out in several steps. We suppose in what follows that (3.15)-(3.17), (3.19)-(3.23), (3.26)-(3.27), and (3.45) hold and we denote by $c$ a strictly positive generic constant which does not depend on time or on $\mu$, and whose value may change from place to place.

In the first step we consider the following auxiliary problem: find a displacement field $\tilde{\mathbf{u}}_{\mu}:[0, T] \rightarrow V$ and an electric potential field $\tilde{\varphi}_{\mu}:[0, T] \rightarrow W$ such that, for all $t \in[0, T]$,

$$
\begin{aligned}
& \left(\mathscr{A} \boldsymbol{\varepsilon}\left(\tilde{\mathbf{u}}_{\mu}(t)\right), \boldsymbol{\varepsilon}(\mathbf{v})\right)_{\mathscr{H}}+\left(\mathscr{E}^{*} \nabla \tilde{\boldsymbol{\varphi}}_{\mu}(t), \boldsymbol{\varepsilon}(\mathbf{v})\right)_{\mathscr{H}}+j_{\mathrm{ad}}\left(\beta(t), \tilde{\mathbf{u}}_{\mu}(t), \mathbf{v}\right)+\frac{1}{\mu} j_{\mathrm{nc}}\left(\widetilde{\mathbf{u}}_{\mu}(t), \mathbf{v}\right) \\
& \quad=(\mathbf{f}(t), \mathbf{v})_{V} \quad \forall \mathbf{v} \in V, \\
& \left(\mathscr{B} \nabla \tilde{\varphi}_{\mu}(t), \nabla \psi\right)_{L^{2}(\Omega)^{d}}-\left(\mathscr{E} \boldsymbol{\varepsilon}\left(\widetilde{\mathbf{u}}_{\mu}(t)\right), \nabla \psi\right)_{L^{2}(\Omega)^{d}}=(q(t), \psi)_{W} \quad \forall \psi \in W .
\end{aligned}
$$

This problem is of the form (4.26), since here $\beta=\beta(t)$ is known, taken from the Signorini Problem 3.1. Therefore, by using the arguments in step (i) of the proof of Theorem 3.5, we deduce that, for each $\mu>0$, problem (5.1)-(5.2) has a unique solution which satisfies $\tilde{\mathbf{u}}_{\mu} \in W^{1, \infty}(0, T ; V), \tilde{\varphi}_{\mu} \in W^{1, \infty}(0, T ; W)$.

We have the following convergence result. 
Lemma 5.1. As $\mu \rightarrow 0$,

$$
\left\|\tilde{\mathbf{u}}_{\mu}(t)-\mathbf{u}(t)\right\|_{V}+\left\|\tilde{\varphi}_{\mu}(t)-\varphi(t)\right\|_{W} \longrightarrow 0,
$$

for all $t \in[0, T]$.

Proof. Let $t \in[0, T]$. We choose $\mathbf{v}=\widetilde{\mathbf{u}}_{\mu}(t)$ in (5.1) and $\psi=\widetilde{\varphi}_{\mu}(t)$ in (5.2) to find

$$
\begin{gathered}
\left(\mathscr{A} \boldsymbol{\varepsilon}\left(\tilde{\mathbf{u}}_{\mu}(t)\right), \boldsymbol{\varepsilon}\left(\widetilde{\mathbf{u}}_{\mu}(t)\right)\right)_{\mathscr{H}}+\left(\mathscr{E} * \nabla \tilde{\varphi}_{\mu}(t)\right), \boldsymbol{\varepsilon}\left(\tilde{\mathbf{u}}_{\mu}(t)\right)_{\mathscr{H}} \\
+j_{\mathrm{ad}}\left(\beta(t), \widetilde{\mathbf{u}}_{\mu}(t), \tilde{\mathbf{u}}_{\mu}(t)\right)+\frac{1}{\mu} j_{\mathrm{nc}}\left(\widetilde{\mathbf{u}}_{\mu}(t), \widetilde{\mathbf{u}}_{\mu}(t)\right) \\
=\left(\mathbf{f}(t), \tilde{\mathbf{u}}_{\mu}(t)\right)_{V}, \\
\left(\mathscr{B} \nabla \tilde{\varphi}_{\mu}(t), \nabla \tilde{\varphi}_{\mu}(t)\right)_{L^{2}(\Omega)^{d}}-\left(\mathscr{E} \boldsymbol{\varepsilon}\left(\tilde{\mathbf{u}}_{\mu}(t)\right), \nabla \tilde{\varphi}_{\mu}(t)\right)_{L^{2}(\Omega)^{d}}=\left(q(t), \tilde{\varphi}_{\mu}(t)\right)_{W} .
\end{gathered}
$$

We add equalities (5.4), (5.5) and use (3.18), (3.52), and (3.57) to obtain

$$
\left(\mathscr{A} \boldsymbol{\varepsilon}\left(\widetilde{\mathbf{u}}_{\mu}(t)\right), \boldsymbol{\varepsilon}\left(\widetilde{\mathbf{u}}_{\mu}(t)\right)\right)_{\mathscr{H}}+\left(\mathscr{B} \nabla \tilde{\varphi}_{\mu}(t), \nabla \tilde{\varphi}_{\mu}(t)\right)_{L^{2}(\Omega)^{d}} \leq\left(\mathbf{f}(t), \widetilde{\mathbf{u}}_{\mu}(t)\right)_{V}+\left(q(t) \tilde{\varphi}_{\mu}(t)\right)_{W}
$$

and, keeping in mind (3.15), (3.17), we deduce that there exists $c>0$ such that

$$
\left\|\tilde{\mathbf{u}}_{\mu}(t)\right\|_{V}+\left\|\tilde{\varphi}_{\mu}(t)\right\|_{W} \leq c\left(\|\mathbf{f}(t)\|_{V}+\|q(t)\|_{W}\right) .
$$

Thus, there exist $\widetilde{\mathbf{u}}(t) \in V, \tilde{\varphi}_{\mu}(t) \in W$, and subsequences of the sequences $\left\{\widetilde{\mathbf{u}}_{\mu}(t)\right\}_{\mu}$, $\left\{\tilde{\varphi}_{\mu}(t)\right\}_{\mu}$, denoted again by $\left\{\tilde{\mathbf{u}}_{\mu}(t)\right\}_{\mu},\left\{\tilde{\varphi}_{\mu}(t)\right\}_{\mu}$, such that

$$
\tilde{\mathbf{u}}_{\mu}(t) \longrightarrow \widetilde{\mathbf{u}}(t) \quad \text { weakly in } V, \quad \tilde{\varphi}_{\mu}(t) \longrightarrow \tilde{\varphi}(t) \quad \text { weakly in } W, \quad \text { as } \mu \longrightarrow 0 .
$$

Using again (3.52), (5.4), and (5.7), after some computations, we find that

$$
j_{\mathrm{nc}}\left(\tilde{\mathbf{u}}_{\mu}(t), \widetilde{\mathbf{u}}_{\mu}(t)\right) \leq c \mu
$$

It follows from (5.8) that

$$
\widetilde{\mathbf{u}}_{\mu}(t) \longrightarrow \tilde{\mathbf{u}}(t) \quad \text { in } L^{2}\left(\Gamma_{3}\right)^{d}, \text { as } \mu \longrightarrow 0
$$

and, recalling (3.30) for $j_{\mathrm{nc}}$ and using (5.9) and (5.10), we find that

$$
\int_{\Gamma_{3}} p_{\nu}\left(\tilde{u}_{\nu}(t)\right) \tilde{u}_{\nu}(t) d s=0
$$

Since the integrand is nonnegative (see (3.19c) and (3.19e)), from the previous equality we find that $p_{v}\left(\tilde{u}_{v}(t)\right) \tilde{u}_{v}(t)=0$ a.e. on $\Gamma_{3}$. It follows now from $(3.45)$ that $\tilde{u}_{v}(t) \leq 0$ a.e. on $\Gamma_{3}$ which shows that $\tilde{\mathbf{u}}(t) \in U$. 
Next, by choosing the test function $\mathbf{v}-\widetilde{\mathbf{u}}_{\mu}(t)$ in (5.1) and keeping in mind the properties of the normal compliance function $p_{\gamma}$, we obtain

$$
\begin{aligned}
& \left(\mathscr{A} \boldsymbol{\varepsilon}\left(\tilde{\mathbf{u}}_{\mu}(t)\right), \boldsymbol{\varepsilon}\left(\mathbf{v}-\tilde{\mathbf{u}}_{\mu}(t)\right)\right)_{\mathscr{H}}+\left(\mathscr{E} * \nabla \tilde{\varphi}_{\mu}(t), \boldsymbol{\varepsilon}\left(\mathbf{v}-\tilde{\mathbf{u}}_{\mu}(t)\right)\right)_{\mathscr{H}}+j_{\mathrm{ad}}\left(\beta(t), \tilde{\mathbf{u}}_{\mu}(t), \mathbf{v}-\tilde{\mathbf{u}}_{\mu}(t)\right) \\
& \quad \geq\left(\mathbf{f}(t), \mathbf{v}-\tilde{\mathbf{u}}_{\mu}(t)\right)_{V} \quad \forall \mathbf{v} \in U .
\end{aligned}
$$

We then test in (5.2) with $\psi-\tilde{\varphi}_{\mu}(t)$, add the resulting equality to (5.12), and use (3.18) to obtain

$$
\begin{aligned}
\left(\mathscr{A} \boldsymbol{\varepsilon}\left(\tilde{\mathbf{u}}_{\mu}(t)\right), \boldsymbol{\varepsilon}\left(\mathbf{v}-\widetilde{\mathbf{u}}_{\mu}(t)\right)\right)_{\mathscr{H}}+\left(\mathscr{B} \nabla \tilde{\varphi}_{\mu}(t), \nabla\left(\psi-\tilde{\varphi}_{\mu}(t)\right)\right)_{L^{2}(\Omega)^{d}} \\
\quad+\left(\mathscr{E} * \nabla \tilde{\varphi}_{\mu}(t), \boldsymbol{\varepsilon}(\mathbf{v})\right)_{\mathscr{H}}-\left(\mathscr{E} \boldsymbol{\varepsilon}\left(\widetilde{\mathbf{u}}_{\mu}(t)\right), \nabla \psi\right)_{L^{2}(\Omega)^{d}}+j_{\mathrm{ad}}\left(\beta(t), \widetilde{\mathbf{u}}_{\mu}(t), \mathbf{v}-\widetilde{\mathbf{u}}_{\mu}(t)\right) \\
\geq\left(\mathbf{f}(t), \mathbf{v}-\widetilde{\mathbf{u}}_{\mu}(t)\right)_{V}+\left(q(t), \psi-\tilde{\varphi}_{\mu}(t)\right)_{W} \quad \forall \mathbf{v} \in U, \psi \in W .
\end{aligned}
$$

Next, we use (3.29), (5.10), and the properties of $R_{v}, \mathbf{R}_{\tau}$, and $p_{\tau}$ to see that

$$
j_{\mathrm{ad}}\left(\beta(t), \tilde{\mathbf{u}}_{\mu}(t), \mathbf{v}-\widetilde{\mathbf{u}}_{\mu}(t)\right) \longrightarrow j_{\mathrm{ad}}(\beta(t), \tilde{\mathbf{u}}(t), \mathbf{v}-\widetilde{\mathbf{u}}(t)) \quad \text { as } \mu \longrightarrow 0,
$$

for any $\mathbf{v} \in V$. Therefore, by (5.8), (5.13), (5.14), and a lower semicontinuity argument we find that

$$
\begin{aligned}
(\mathscr{A} \boldsymbol{\varepsilon}(\tilde{\mathbf{u}}(t)), \boldsymbol{\varepsilon}(\mathbf{v}-\tilde{\mathbf{u}}(t)))_{\mathscr{H}}+(\mathscr{B} \nabla \tilde{\varphi}(t), \nabla(\psi-\tilde{\varphi}(t)))_{L^{2}(\Omega)^{d}} \\
\quad+(\mathscr{E} * \nabla \tilde{\varphi}(t), \boldsymbol{\varepsilon}(\mathbf{v}))_{\mathscr{H}}-(\mathscr{E} \boldsymbol{\varepsilon}(\tilde{\mathbf{u}}(t)), \nabla \psi)_{L^{2}(\Omega)^{d}}+j_{\mathrm{ad}}(\beta(t), \tilde{\mathbf{u}}(t), \mathbf{v}-\tilde{\mathbf{u}}(t)) \\
\geq(\mathbf{f}(t), \mathbf{v}-\tilde{\mathbf{u}}(t))_{V}+(q(t), \psi-\tilde{\varphi}(t))_{W} \quad \forall \mathbf{v} \in U, \psi \in W .
\end{aligned}
$$

We take now $\mathbf{v}=\mathbf{u}(t)$ and $\psi=\varphi(t)$ in (5.15) to obtain

$$
\begin{aligned}
(\mathscr{A} \boldsymbol{\varepsilon}(\tilde{\mathbf{u}}(t)), \boldsymbol{\varepsilon}(\mathbf{u}(t)-\tilde{\mathbf{u}}(t)))_{\mathscr{H}}+(\mathscr{B} \nabla \tilde{\varphi}(t), \nabla(\varphi(t)-\tilde{\varphi}(t)))_{L^{2}(\Omega)^{d}} \\
\quad+(\mathscr{E} * \nabla \tilde{\varphi}(t), \boldsymbol{\varepsilon}(\mathbf{u}(t)))_{\mathscr{H}}-(\mathscr{E} \boldsymbol{\varepsilon}(\tilde{\mathbf{u}}(t)), \nabla \varphi(t))_{L^{2}(\Omega)^{d}}+j_{\mathrm{ad}}(\beta(t), \tilde{\mathbf{u}}(t), \mathbf{u}(t)-\widetilde{\mathbf{u}}(t)) \\
\geq(\mathbf{f}(t), \mathbf{u}(t)-\tilde{\mathbf{u}}(t))_{V}+(q(t), \varphi(t)-\tilde{\varphi}(t))_{W} \quad \forall \mathbf{v} \in U, \psi \in W .
\end{aligned}
$$

On the other hand, we take $\mathbf{v}=\widetilde{\mathbf{u}}(t)$ in (3.31) and add the resulting inequality to the equality (3.32) in which we take $\psi=\widetilde{\varphi}(t)-\varphi(t)$; as a result we obtain

$$
\begin{aligned}
(\mathscr{A} \boldsymbol{\varepsilon}(\mathbf{u}(t)), \boldsymbol{\varepsilon}(\tilde{\mathbf{u}}(t)-\mathbf{u}(t)))_{\mathscr{H}}+(\mathscr{B} \nabla \varphi(t), \nabla(\tilde{\varphi}(t)-\varphi(t)))_{L^{2}(\Omega)^{d}} \\
\quad+(\mathscr{E} * \nabla \varphi(t), \boldsymbol{\varepsilon}(\tilde{\mathbf{u}}(t))-\mathbf{u}(t))_{\mathscr{H}} \\
\quad-(\mathscr{E} \boldsymbol{\varepsilon}(\mathbf{u}(t)), \nabla(\tilde{\varphi}(t)-\varphi(t)))_{L^{2}(\Omega)^{d}}+j_{\mathrm{ad}}(\beta(t), \mathbf{u}(t), \tilde{\mathbf{u}}(t)-\mathbf{u}(t)) \\
\geq \\
(\mathbf{f}(t), \tilde{\mathbf{u}}(t)-\mathbf{u}(t))_{V}+(q(t), \tilde{\varphi}(t)-\varphi(t))_{W} \quad \forall \mathbf{v} \in U, \psi \in W .
\end{aligned}
$$


We add (5.16), (5.17) and use again (3.15), (3.17), and (3.18) to see that

$$
\begin{aligned}
& c\left(\left\|\tilde{\mathbf{u}}_{\mu}(t)-\mathbf{u}(t)\right\|_{V}^{2}+\left\|\tilde{\varphi}_{\mu}(t)-\varphi(t)\right\|_{W}^{2}\right) \\
& \quad \leq j_{\mathrm{ad}}(\beta(t), \tilde{\mathbf{u}}(t), \mathbf{u}(t)-\tilde{\mathbf{u}}(t))+j_{\mathrm{ad}}(\beta(t), \mathbf{u}(t), \tilde{\mathbf{u}}(t)-\mathbf{u}(t)) .
\end{aligned}
$$

We now use (3.51) to show that the right-hand side of this inequality is nonpositive and, thus,

$$
\tilde{\mathbf{u}}(t)=\mathbf{u}(t), \quad \tilde{\varphi}_{\mu}(t)=\varphi(t) .
$$

We conclude that $\mathbf{u}(t)$ is the unique weak limit in $V$ of any subsequence of the sequence $\left\{\tilde{\mathbf{u}}_{\mu}(t)\right\}_{\mu}$ and, therefore, we find that the whole sequence $\left\{\tilde{\mathbf{u}}_{\mu}(t)\right\}_{\mu}$ converges weakly to the element $\mathbf{u}(t) \in U$. The same argument shows that the whole sequence $\left\{\varphi_{\mu}(t)\right\}_{\mu}$ converges weakly to the element $\varphi(t) \in W$.

Using now (5.13) with $\mathbf{v}=\mathbf{u}(t), \psi=\varphi(t)$, and assumptions (3.15), (3.17) on $\mathscr{A}, \mathscr{B}$, we deduce that

$$
\begin{aligned}
c\left(\| \widetilde{\mathbf{u}}_{\mu}(\right. & \left.t)-\mathbf{u}(t)\left\|_{V}^{2}+\right\| \widetilde{\varphi}_{\mu}(t)-\varphi(t) \|_{W}^{2}\right) \\
\leq & \left(\mathbf{f}(t), \widetilde{\mathbf{u}}_{\mu}(t)-\mathbf{u}(t)\right)_{V}+\left(q(t), \widetilde{\varphi}_{\mu}(t)-\varphi(t)\right)_{W}+j_{\mathrm{ad}}\left(\beta(t), \widetilde{\mathbf{u}}_{\mu}(t), \mathbf{u}(t)\right)-\widetilde{\mathbf{u}}_{\mu}(t) \\
& +\left(\mathscr{A} \boldsymbol{\varepsilon}(\mathbf{u}(t)), \boldsymbol{\varepsilon}\left(\mathbf{u}(t)-\widetilde{\mathbf{u}}_{\mu}(t)\right)\right)_{\mathscr{H}}+\left(\mathscr{B} \nabla \varphi(t), \nabla\left(\varphi(t)-\widetilde{\varphi}_{\mu}(t)\right)\right)_{L^{2}(\Omega)^{d}} \\
& +\left(\mathscr{E} * \nabla \widetilde{\varphi}_{\mu}(t), \boldsymbol{\varepsilon}(\mathbf{u})\right)_{\mathscr{H}}-\left(\mathscr{E} \boldsymbol{\varepsilon}\left(\widetilde{\mathbf{u}}_{\mu}(t)\right), \nabla \varphi\right)_{L^{2}(\Omega)^{d}} .
\end{aligned}
$$

Taking into account (3.18), (5.8), (5.14), and (5.19), we obtain from the previous inequality that

$$
\left\|\tilde{\mathbf{u}}_{\mu}(t)-\mathbf{u}(t)\right\|_{V}^{2}+\left\|\tilde{\varphi}_{\mu}(t)-\varphi(t)\right\|_{W}^{2} \longrightarrow 0 \quad \text { as } \mu \longrightarrow 0,
$$

which concludes the proof.

The next step in the proof of the theorem is the following.

Lemma 5.2. There exists $c>0$ such that

$$
\left\|\tilde{\mathbf{u}}_{\mu}(t)-\mathbf{u}_{\mu}(t)\right\|_{V}+\left\|\tilde{\varphi}_{\mu}(t)-\varphi_{\mu}(t)\right\|_{W} \leq c\left\|\beta_{\mu}(t)-\beta(t)\right\|_{L^{2}\left(\Gamma_{3}\right)},
$$

for all $t \in[0, T]$ and $\mu>0$.

Proof. Let $t \in[0, T]$ and $\mu>0$. From (3.35), (3.36), (5.1), and (5.2) after some computations we find that

$$
\begin{aligned}
(\mathscr{A} \boldsymbol{\varepsilon} & \left.\left(\mathbf{u}_{\mu}(t)-\tilde{\mathbf{u}}_{\mu}(t)\right), \boldsymbol{\varepsilon}\left(\tilde{\mathbf{u}}_{\mu}(t)-\mathbf{u}_{\mu}(t)\right)\right)_{\mathscr{H}} \\
& +\left(\mathscr{B} \nabla\left(\varphi_{\mu}(t)-\tilde{\varphi}_{\mu}(t)\right), \nabla\left(\tilde{\varphi}_{\mu}(t)-\varphi_{\mu}(t)\right)\right)_{L^{2}(\Omega)^{d}} \\
& +j_{\mathrm{ad}}\left(\beta_{\mu}(t), \mathbf{u}_{\mu}(t), \tilde{\mathbf{u}}_{\mu}(t)-\mathbf{u}_{\mu}(t)\right)-j_{\mathrm{ad}}\left(\beta(t), \tilde{\mathbf{u}}_{\mu}(t), \tilde{\mathbf{u}}_{\mu}(t)-\mathbf{u}_{\mu}(t)\right) \\
& +\frac{1}{\mu} j_{\mathrm{nc}}\left(\mathbf{u}_{\mu}(t), \tilde{\mathbf{u}}_{\mu}(t)-\mathbf{u}_{\mu}(t)\right)-\frac{1}{\mu} j_{\mathrm{nc}}\left(\tilde{\mathbf{u}}_{\mu}(t), \tilde{\mathbf{u}}_{\mu}(t)-\mathbf{u}_{\mu}(t)\right)=0 .
\end{aligned}
$$


We use (3.47), (3.49), and (3.56) to see that

$$
\begin{gathered}
j_{\mathrm{ad}}\left(\beta_{\mu}(t), \mathbf{u}_{\mu}(t), \widetilde{\mathbf{u}}_{\mu}(t)-\mathbf{u}_{\mu}(t)\right)-j_{\mathrm{ad}}\left(\beta(t), \widetilde{\mathbf{u}}_{\mu}(t), \widetilde{\mathbf{u}}_{\mu}(t)-\mathbf{u}_{\mu}(t)\right) \\
\quad \leq c\left\|\beta_{\mu}(t)-\beta(t)\right\|_{L^{2}\left(\Gamma_{3}\right)}\left\|\tilde{\mathbf{u}}_{\mu}(t)-\mathbf{u}_{\mu}(t)\right\|_{V}, \\
j_{\mathrm{nc}}\left(\mathbf{u}_{\mu}(t), \tilde{\mathbf{u}}_{\mu}(t)-\mathbf{u}_{\mu}(t)\right)-j_{\mathrm{nc}}\left(\tilde{\mathbf{u}}_{\mu}(t), \tilde{\mathbf{u}}_{\mu}(t)-\mathbf{u}_{\mu}(t)\right) \leq 0 .
\end{gathered}
$$

We use now the last two inequalities in (5.23) and combine the resulting inequality with (3.15), (3.17) to obtain (5.22).

We have now all the ingredients to prove Theorem 3.6.

Proof. Let $t \in[0, T]$. Using norm triangle inequality and (5.22), we obtain

$$
\begin{aligned}
& \left\|\mathbf{u}_{\mu}(t)-\mathbf{u}(t)\right\|_{V}+\left\|\varphi_{\mu}(t)-\varphi(t)\right\|_{W}+\left\|\beta_{\mu}(t)-\beta(t)\right\|_{L^{2}\left(\Gamma_{3}\right)} \\
& \quad \leq\left\|\tilde{\mathbf{u}}_{\mu}(t)-\mathbf{u}(t)\right\|_{V}+\left\|\tilde{\varphi}_{\mu}(t)-\varphi(t)\right\|_{W}+\left.c\left\|\beta_{\mu}(t)-\beta(t)\right\|\right|_{L^{2}\left(\Gamma_{3}\right)} .
\end{aligned}
$$

We now use (3.33), (3.34), (3.37), (3.38) and arguments similar to those used in the proof of (4.19) to see that

$$
\left\|\beta_{\mu}(t)-\beta(t)\right\|_{L^{2}\left(\Gamma_{3}\right)} \leq c \int_{0}^{t}\left\|\mathbf{u}_{\mu}(s)-\mathbf{u}(s)\right\|_{V} d s .
$$

Plugging (5.26) in (5.25) and applying the Gronwall lemma to the resulting inequality, we obtain

$$
\begin{aligned}
& \left\|\mathbf{u}_{\mu}(t)-\mathbf{u}(t)\right\|_{V}+\left\|\varphi_{\mu}(t)-\varphi(t)\right\|_{W}+\left\|\beta_{\mu}(t)-\beta(t)\right\|_{L^{2}\left(\Gamma_{3}\right)} \\
& \quad \leq\left\|\tilde{\mathbf{u}}_{\mu}(t)-\mathbf{u}(t)\right\|_{V}+\left\|\tilde{\varphi}_{\mu}(t)-\varphi(t)\right\|_{W}+c \int_{0}^{t}\left(\left\|\tilde{\mathbf{u}}_{\mu}(s)-\mathbf{u}(s)\right\|_{V}+\left\|\tilde{\varphi}_{\mu}(s)-\varphi(s)\right\|_{W}\right) d s .
\end{aligned}
$$

On the other hand, we note that (5.7) implies that

$$
\left\|\tilde{\mathbf{u}}_{\mu}(t)-\mathbf{u}(t)\right\|_{V}+\left\|\tilde{\varphi}_{\mu}(t)-\varphi(t)\right\|_{W} \leq c\left(\|\mathbf{f}(t)\|_{V}+\|q(t)\|_{W}+\|\mathbf{u}(t)\|_{V}+\|\varphi(t)\|_{W}\right) .
$$

Since (5.3) and (5.28) hold for all $t \in[0, T]$, from Lebesgue convergence theorem we deduce that

$$
\int_{0}^{t}\left(\left\|\tilde{\mathbf{u}}_{\mu}(s)-\mathbf{u}(s)\right\|_{V}+\left\|\tilde{\varphi}_{\mu}(s)-\varphi(s)\right\|_{W}\right) d s \longrightarrow 0 \quad \text { as } \mu \longrightarrow 0 .
$$

The convergence result (3.46) is now a consequence of (5.3), (5.27), and (5.29).

We end this section with the remark that Theorem 3.6 implies the following convergence result, in terms of stress and electric displacement field:

$$
\left\|\boldsymbol{\sigma}_{\mu}(t)-\boldsymbol{\sigma}(t)\right\|_{\mathscr{H}_{1}}+\left\|\mathbf{D}_{\mu}(t)-\mathbf{D}(t)\right\|_{\mathscr{W}_{1}} \longrightarrow 0
$$


as $\mu \rightarrow 0$, for all $t \in[0, T]$. Indeed, let $t \in[0, T]$ and $\mu>0$; the electroelastic constitutive laws (2.1), (2.2), (2.17), and (2.18) combined with the properties of the operators $\mathscr{A}$ and $\mathscr{B}$ yield

$$
\left\|\boldsymbol{\sigma}_{\mu}(t)-\boldsymbol{\sigma}(t)\right\|_{\mathscr{H}}+\left\|\mathbf{D}_{\mu}(t)-\mathbf{D}(t)\right\|_{L^{2}(\Omega)^{d}} \leq c\left(\left\|\mathbf{u}_{\mu}(t)-\mathbf{u}(t)\right\|_{V}+\left\|\varphi_{\mu}(t)-\varphi(t)\right\|_{W}\right) .
$$

Also, since (3.31) and (3.35) imply $\operatorname{Div} \boldsymbol{\sigma}_{\mu}(t)=\operatorname{Div} \boldsymbol{\sigma}(t)=-\mathbf{f}_{0}(t)$ and (3.32) and (3.36) imply $\operatorname{div} \mathbf{D}_{\mu}(t)=\operatorname{div} \mathbf{D}(t)=-q_{0}(t)$, we deduce that

$$
\begin{gathered}
\left\|\boldsymbol{\sigma}_{\mu}(t)-\boldsymbol{\sigma}(t)\right\|_{\mathscr{H}_{1}}=\left\|\boldsymbol{\sigma}_{\mu}(t)-\boldsymbol{\sigma}(t)\right\|_{\mathscr{H}}, \\
\left\|\mathbf{D}_{\mu}(t)-\mathbf{D}(t)\right\|_{\mathscr{W}_{1}}=\left\|\mathbf{D}_{\mu}(t)-\mathbf{D}(t)\right\|_{L^{2}(\Omega)^{d}} .
\end{gathered}
$$

The convergence (5.30) is now a consequence of (3.46), (5.31), and (5.32).

\section{References}

[1] K. T. Andrews, L. Chapman, J. R. Fernández, M. Fisackerly, M. Shillor, L. Vanerian, and T. VanHouten, A membrane in adhesive contact, SIAM Journal on Applied Mathematics 64 (2003), no. $1,152-169$.

[2] K. T. Andrews and M. Shillor, Dynamic adhesive contact of a membrane, Advances in Mathematical Sciences and Applications 13 (2003), no. 1, 343-356.

[3] R. C. Batra and J. S. Yang, Saint-Venant's principle in linear piezoelectricity, Journal of Elasticity 38 (1995), no. 2, 209-218.

[4] P. Bisegna, F. Lebon, and F. Maceri, The unilateral frictional contact of a piezoelectric body with a rigid support, Contact Mechanics (Praia da Consolação, 2001) (J. A. C. Martins and M. D. P. Monteiro Marques, eds.), Solid Mech. Appl., vol. 103, Kluwer Academic, Dordrecht, 2002, pp. 347-354.

[5] T. Buchukuri and T. Gegelia, Some dynamic problems of the theory of electroelasticity, Memoirs on Differential Equations and Mathematical Physics 10 (1997), 1-53.

[6] O. Chau, J. R. Fernández, M. Shillor, and M. Sofonea, Variational and numerical analysis of a quasistatic viscoelastic contact problem with adhesion, Journal of Computational and Applied Mathematics 159 (2003), no. 2, 431-465.

[7] O. Chau, M. Shillor, and M. Sofonea, Dynamic frictionless contact with adhesion, Zeitschrift für Angewandte Mathematik und Physik 55 (2004), no. 1, 32-47.

[8] M. Cocu and R. Rocca, Existence results for unilateral quasistatic contact problems with friction and adhesion, Mathematical Modelling and Numerical Analysis 34 (2000), no. 5, 981-1001.

[9] J. R. Fernández, M. Shillor, and M. Sofonea, Analysis and numerical simulations of a dynamic contact problem with adhesion, Mathematical and Computer Modelling 37 (2003), no. 12-13, $1317-1333$.

[10] M. Frémond, Équilibre de structures qui adhèrent à leur support, Comptes Rendus des Séances de l'Académie des Sciences. Série II 295 (1982), no. 11, 913-916.

[11] _ Adhérence des solides, Journal de Mécanique Théorique et Appliquée 6 (1987), no. 3, 383-407.

[12] N_ Non-Smooth Thermomechanics, Springer, Berlin, 2002.

[13] W. Han, K. L. Kuttler, M. Shillor, and M. Sofonea, Elastic beam in adhesive contact, International Journal of Solids and Structures 39 (2002), no. 5, 1145-1164.

[14] W. Han and M. Sofonea, Quasistatic Contact Problems in Viscoelasticity and Viscoplasticity, AMS/IP Studies in Advanced Mathematics, vol. 30, American Mathematical Society, Rhode Island; International Press, Massachusetts, 2002. 
[15] T. Ikeda, Fundamentals of Piezoelectricity, Oxford University Press, Oxford, 1990.

[16] F. Maceri and P. Bisegna, The unilateral frictionless contact of a piezoelectric body with a rigid support, Mathematical and Computer Modelling 28 (1998), no. 4-8, 19-28.

[17] M. Raous, L. Cangémi, and M. Cocu, A consistent model coupling adhesion, friction, and unilateral contact, Computer Methods in Applied Mechanics and Engineering 177 (1999), no. 3-4, 383399.

[18] J. Rojek and J. J. Telega, Contact problems with friction, adhesion and wear in orthopaedic biomechanics. I: general developments, Journal of Theoretical and Applied Mechanics 39 (2001), 655677.

[19] J. Rojek, J. J. Telega, and S. Stupkiewicz, Contact problems with friction, adhesion and wear in orthopaedic biomechanics. II: numerical implementation and application to implanted knee joints, Journal of Theoretical and Applied Mechanics 39 (2001), 679-706.

[20] M. Shillor, M. Sofonea, and J. J. Telega, Models and Variational Analysis of Quasistatic Contact, Lecture Notes in Physics, vol. 655, Springer, Berlin, 2004.

[21] M. Sofonea and El-H. Essoufi, A piezoelectric contact problem with slip dependent coefficient of friction, Mathematical Modelling and Analysis 9 (2004), no. 3, 229-242.

[22] _ Quasistatic frictional contact of a viscoelastic piezoelectric body, Advances in Mathematical Sciences and Applications 14 (2004), no. 2, 613-631.

[23] M. Sofonea, W. Han, and M. Shillor, Analysis and Approximation of Contact Problems with Adhesion or Damage, Pure and Applied Mathematics (Boca Raton), vol. 276, Chapman \& Hall/CRC, Florida, 2006.

[24] M. Sofonea and T.-V. Hoarau-Mantel, Elastic frictionless contact problems with adhesion, Advances in Mathematical Sciences and Applications 15 (2005), no. 1, 49-68.

[25] C. Talon and A. Curnier, A model of adhesion added to contact with friction, Contact Mechanics (Praia da Consolação, 2001) (J. A. C. Martins and M. D. P. Monteiro Marques, eds.), Solid Mech. Appl., vol. 103, Kluwer Academic, Dordrecht, 2002, pp. 161-168.

Mircea Sofonea: Laboratoire de Mathématiques et Physique pour les Systèmes, Université de Perpignan, 52 avenue Paul Alduy, 66860 Perpignan, France

E-mail address: sofonea@univ-perp.fr

Rachid Arhab: Laboratoire de Mathématiques et Physique pour les Systèmes, Université de Perpignan, 52 avenue Paul Alduy, 66860 Perpignan, France

E-mail address: rachid.arhab@univ-perp.fr

Raafat Tarraf: Laboratoire de Mathématiques et Physique pour les Systèmes, Université de Perpignan, 52 avenue Paul Alduy, 66860 Perpignan, France

E-mail address: raafat.tarraf@univ-perp.fr 


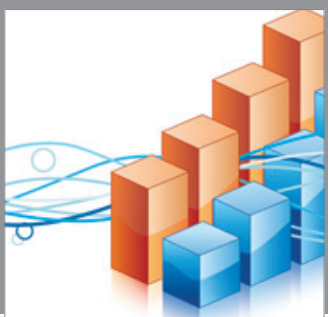

Advances in

Operations Research

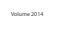

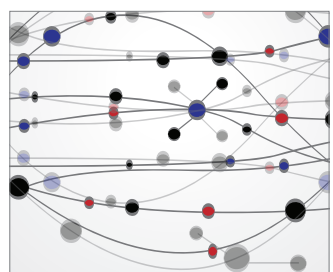

\section{The Scientific} World Journal
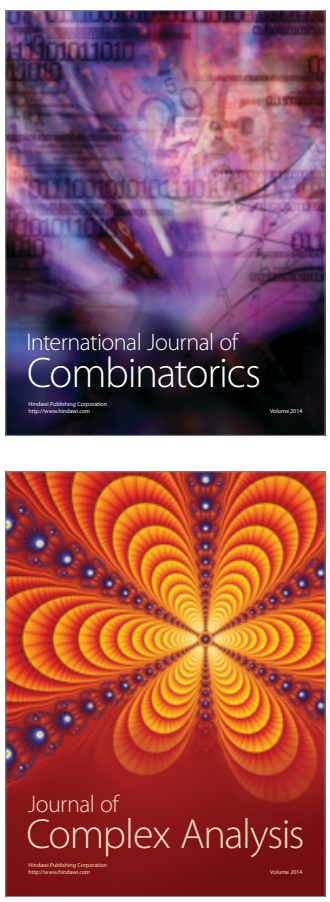

International Journal of

Mathematics and

Mathematical

Sciences
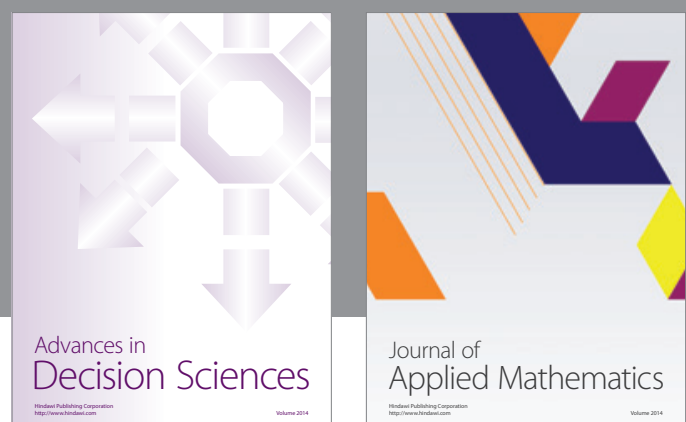

Journal of

Applied Mathematics
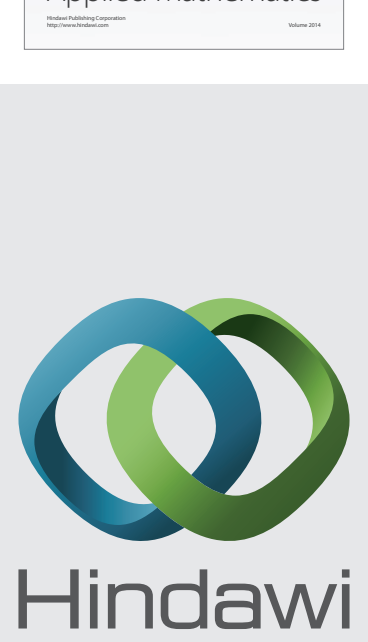

Submit your manuscripts at http://www.hindawi.com
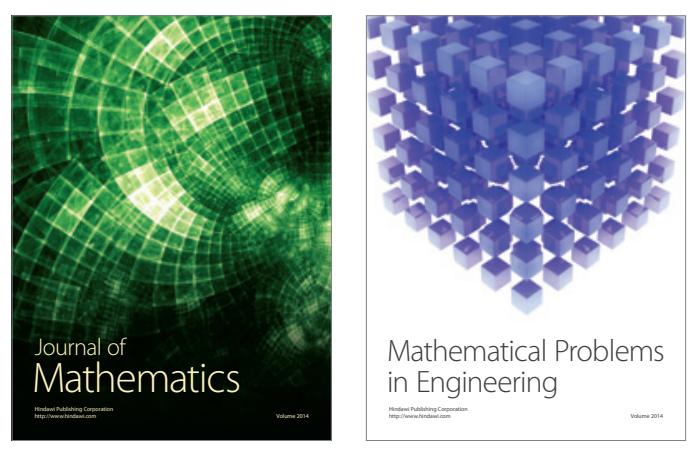

Mathematical Problems in Engineering
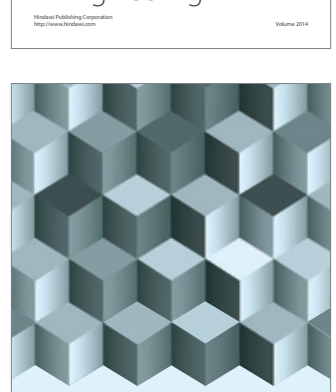

Journal of

Function Spaces
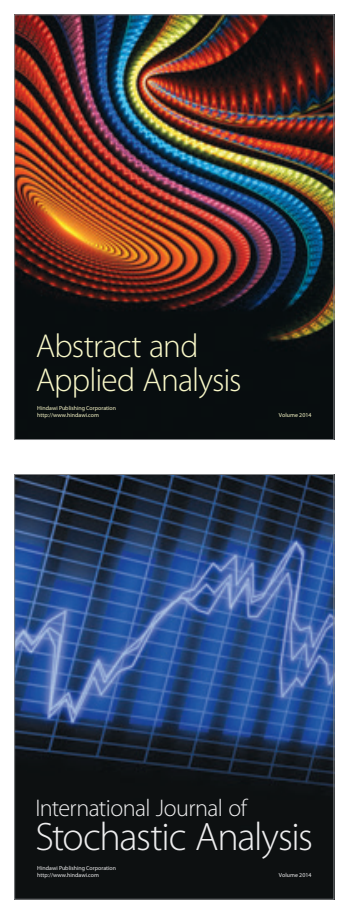

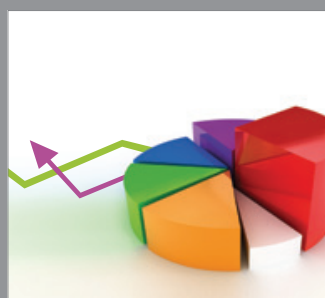

ournal of

Probability and Statistics

Promensencen
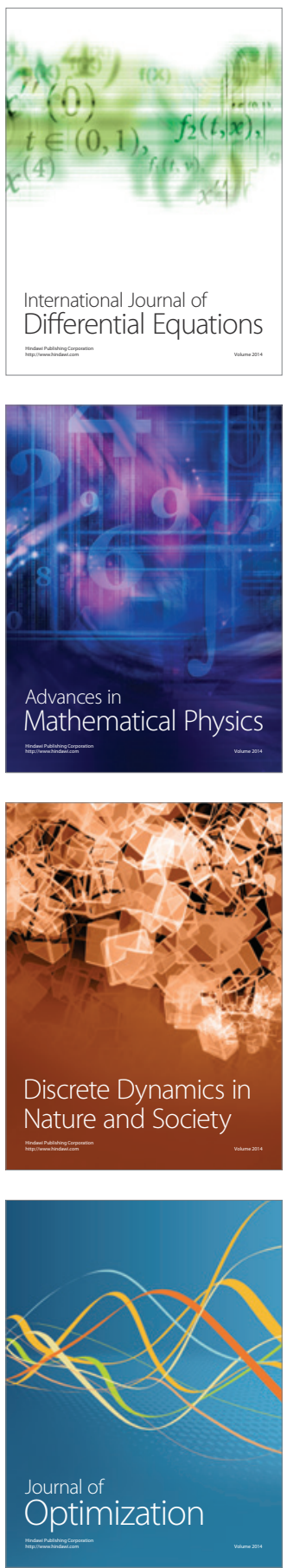